\title{
Computational Methods in Organic Thermochemistry. II. Enthalpies and Free Energies of Formation for Functional Derivatives of Organic \\ Hydrocarbons
}

\author{
Douglas Bond
}

Contribution from the Computer Chemie Centrum, Friedrich-Alexander-Universität, Erlangen-

Nürnberg, Nägelsbach Strasse 25, 91052 Erlangen, Germany

\section{Supporting Information (18 pages)}

Full citation for Reference 13

Gaussian 98, Revision A.11.3, M. J. Frisch, G. W. Trucks, H. B. Schlegel, G. E. Scuseria, M. A. Robb, J. R. Cheeseman, V. G. Zakrzewski, J. A. Montgomery, Jr., R. E. Stratmann, J. C. Burant, S. Dapprich, J. M. Millam, A. D. Daniels, K. N. Kudin, M. C. Strain, O. Farkas, J. Tomasi, V. Barone, M. Cossi, R. Cammi, B. Mennucci, C. Pomelli, C. Adamo, S. Clifford, J. Ochterski, G. A. Petersson, P. Y. Ayala, Q. Cui, K. Morokuma, N. Rega, P. Salvador, J. J. Dannenberg, D. K. Malick, A. D. Rabuck, K. Raghavachari, J. B. Foresman, J. Cioslowski, J. V. Ortiz, A. G. Baboul, B. B. Stefanov, G. Liu, A. Liashenko, P. Piskorz, I. Komaromi, R. Gomperts, R. L. Martin, D. J. Fox, T. Keith, M. A. Al-Laham, C. Y. Peng, A. Nanayakkara, M. Challacombe, P. M. W. Gill, B. Johnson, W. Chen, M. W. Wong, J. L. Andres, C. Gonzalez, M. Head-Gordon, E. S. Replogle, and J. A. Pople, Gaussian, Inc., Pittsburgh PA, 2002.

\section{Full citation for Reference 14}

M. J. Frisch, G. W. Trucks, H. B. Schlegel, G. E. Scuseria, M. A. Robb, J. R. Cheeseman, J. A. Montgomery, Jr., T. Vreven, K. N. Kudin, J. C. Burant, J. M. Millam, S. S. Iyengar, J. Tomasi, V. Barone, B. Mennucci, M. Cossi, G. Scalmani, N. Rega, G. A. Petersson, H. Nakatsuji, M. Hada, M. Ehara, K. Toyota, R. Fukuda, J. Hasegawa, M. Ishida, T. Nakajima, Y. Honda, O. Kitao, H. Nakai, M. Klene, X. Li, J. E. Knox, H. P. Hratchian, J. B. Cross, V. Bakken, C. Adamo, J. Jaramillo, R. Gomperts, R. E. Stratmann, O. Yazyev, A. J. Austin, R. Cammi, C. Pomelli, J. W. Ochterski, P. Y. Ayala, K. Morokuma, G. A. Voth, P. Salvador, J. J. Dannenberg, V. G. Zakrzewski, S. Dapprich, A. D. Daniels, M. C. Strain, O. Farkas, D. K. Malick, A. D. Rabuck, K. Raghavachari, J. B. Foresman, J. V. Ortiz, Q. Cui, A. G. Baboul, S. Clifford, J. Cioslowski, B. B. Stefanov, G. Liu, A. Liashenko, P. Piskorz, I. Komaromi, R. L. Martin, D. J. Fox, T. Keith, M. A. Al-Laham, C. Y. Peng, A. Nanayakkara, M.

Challacombe, P. M. W. Gill, B. Johnson, W. Chen, M. W. Wong, C. Gonzalez, and J. A. Pople, Gaussian, Inc., Wallingford CT, 2004.

Table of Contents:

Table S1: Standard state element entropies

Page

Table S2: Experimental energies for inorganic compounds

S2

Table S3: Gas phase atom computed energies for G3, and G3MP2 $\quad$ S2 model chemistries.

Table S4: Input geometries

$$
\text { S3 }
$$

Absolute energies of substances computed for this work is found in the spreadsheet:

Thermochemistry_02.xls, also available via the internet. 
Table S1: Standard state element entropies in $\mathrm{J} \mathrm{mol}^{-1} \mathrm{~K}$

$\begin{array}{lc}\text { Element } & \mathrm{S} \\ \mathrm{C} \text { (graphite) } & 5.686 \\ \mathrm{H}_{2} & 130.68 \\ \mathrm{O}_{2} & 205.15 \\ \mathrm{~N}_{2} & 191.61 \\ \mathrm{Cl}_{2} & 223.08 \\ \mathrm{~S}_{8} & 430.23 \\ \mathrm{~F}_{2} & 202.79 \\ \mathrm{Br}_{2} & 245.47 \\ \mathrm{Si} & 18.82\end{array}$

These are from reference 1 with the exception of the values for graphite and silicon which come from the JANAF Thermochemical Tables, $2^{\text {nd }}$ edition

Table S2: Molecular energies for inorganic compounds Table S2a: Inorganic experimental energies. All values from reference 2 except for the three silicon compounds which are from reference 1 .

\begin{tabular}{lrrc}
\multicolumn{1}{c}{ Compound } & $\Delta \mathrm{H}_{\mathrm{f}}{ }^{\circ}$ & $\Delta \mathrm{G}_{\mathrm{f}}{ }^{\circ}$ & $\mathrm{S}^{\circ}$ \\
Water & -241.83 & -228.58 & 188.84 \\
Carbon Dioxide & -393.51 & -394.38 & 213.79 \\
$\mathrm{NH}_{3}$ & -45.94 & -16.41 & 192.77 \\
$\mathrm{HCN}$ & 132.00 & 121.57 & 201.82 \\
$\mathrm{HCl}$ & -92.3 & -95.29 & 186.90 \\
$\mathrm{H}_{2} \mathrm{~S}$ & -20.60 & -33.48 & 205.81 \\
$\mathrm{H}_{2} \mathrm{~S}_{2}$ & 15.50 & -11.27 & 252.40 \\
$\mathrm{H}_{2} \mathrm{O}_{2}$ & -135.88 & -133.26 & 129.89 \\
$\mathrm{H}_{2} \mathrm{~N}_{2}$ & 211.86 & 242.86 & 218.33 \\
$\mathrm{H}_{2} \mathrm{~N}_{4}$ & 95.18 & 159.14 & 238.46 \\
$\mathrm{SiH}_{4}$ & 34.31 & 56.83 & 204.65 \\
$\mathrm{SiCl}_{4}$ & -662.75 & -622.76 & 330.86 \\
$\mathrm{Si}_{4}\left(\mathrm{CH}_{3}\right)_{4}$ & -233.70 & -43.68 & 361.12
\end{tabular}

Table S3: Gas phase atom computed energies for G3, and G3MP2, model chemistries.

\begin{tabular}{lrrrr} 
& \multicolumn{2}{c}{$G 3$} & \multicolumn{2}{c}{$G 3 M P 2$} \\
Water & $\Delta \mathrm{H}$ & $\Delta G$ & $\Delta \mathrm{H}$ & \multicolumn{1}{c}{$\Delta \mathrm{G}$} \\
Carbon Dioxide & -76.378993 & -76.399436 & -76.339449 & -76.359891 \\
$\mathrm{NH}_{3}$ & -188.495284 & -188.516561 & -188.374474 & -188.395749 \\
$\mathrm{HCN}$ & -56.503212 & -56.525017 & -56.466333 & -56.488138 \\
$\mathrm{HCl}$ & -93.371960 & -93.394754 & -93.295484 & -93.318278 \\
$\mathrm{H}_{2} \mathrm{~S}$ & -460.651364 & -460.672527 & -460.349216 & -460.370379 \\
$\mathrm{H}_{2} \mathrm{~S}_{2}$ & -399.234586 & -399.257897 & -398.940541 & -398.963852 \\
$\mathrm{H}_{2} \mathrm{O}_{2}$ & -797.282213 & -797.310837 & -796.692238 & -796.720861 \\
$\mathrm{H}_{2} \mathrm{~N}_{2}$ & -151.459965 & -151.486371 & -151.378385 & -151.404797 \\
$\mathrm{H}_{2} \mathrm{~N}_{4}$ & -110.568869 & -110.593568 & -110.492610 & -110.517309 \\
$\mathrm{SiH}_{4}$ & -111.771036 & -111.797167 & -111.695402 & -111.721533 \\
$\mathrm{SiCl}_{4}$ & -291.706008 & -291.729240 & -291.426631 & -291.449863 \\
$\mathrm{Si}_{4}\left(\mathrm{CH}_{3}\right)_{4}$ & -2129.782485 & -2129.820338 & -2128.286424 & -2128.324277 \\
& -448.848330 & -448.891948 & -448.421145 & -448.464764
\end{tabular}


Table S3: Input geometries for all compounds not available in the Computational Database of reference 2. There are a few compounds which are found below and in reference 2. In that event, we have used a different starting conformation.

\section{Alcohols}

1,2-Ethanediol

$\mathrm{C}, 0,0.5243,0.2568,-0.7812$

$\mathrm{C}, 0,0.52280 .3034,0.7431$

$\mathrm{H}, 0,1.55006,0.21537,-1.16534$

$\mathrm{H}, 0,0.06241,1.18226,-1.1752$

$\mathrm{O}, 0,-0.15410,-0.89392,-1.24601$

$\mathrm{O}, 0,-0.81728,0.21016,1.23775$

$\mathrm{H}, 0,1.03098,1.20553,1.11580$

$\mathrm{H}, 0,1.03980,-0.5743,1.14190$

$\mathrm{H}, 0,-0.93596,-0.97441,-0.67157$

$\mathrm{H}, 0,-1.25959,1.05353,1.04916$

1-Propanol

$\mathrm{H}, 0,-0.07728,1.59855,-1.42036$ C, $0,-0.07885,1.59956,-0.32289$ $\mathrm{H}, 0,0.96190,1.59011,0.01368$ $\mathrm{C}, 0,-0.83553,0.38685,0.22704$ $\mathrm{H}, 0,-0.5389,2.53696,0.00930$ $\mathrm{C}, 0,-0.2200,-0.94522,-0.1853$ $\mathrm{H}, 0,-1.8786259501,0.40147$,0.11690

$\mathrm{H}, 0,-0.86259,0.42759,1.32360$ $\mathrm{H}, 0,-0.19035,-1.01491,-1.28630$ H, $0,-0.84801,-1.77424,0.18053$ $\mathrm{O}, 0,1.09283,-1.02077,0.36540$ $\mathrm{H}, 0,1.49757,-1.84653,0.06060$

1-Butanol

C, $0,-0.58995,-1.1983,1.99882$ $\mathrm{H}, 0,-0.11707,-2.1388,1.73049$ $\mathrm{H}, 0,-0.32613,-0.97883,3.02881$ $\mathrm{H}, 0,-1.66574,-1.34685,1.95778$ C, $0,-0.14788,-0.07338,1.0635$ $\mathrm{H}, 0,0.92360,0.0715,1.14494$ H, $0,-0.61235,0.85985,1.37716$ $\mathrm{O}, 0,1.33123,0.83773,-1.32881$ C, $0,-0.0714,0.75414,-1.34837$ $\mathrm{H}, 0,-0.42429,0.5321,-2.35395$ $\mathrm{H}, 0,-0.51520,1.70223,-1.04669$ C, $, 0,-0.50691,-0.34780,-0.39761$ $\mathrm{H}, 0,-0.04827,-1.27888,-0.7205$ $\mathrm{H}, 0,-1.58365,-0.47526,-0.49441$ $\mathrm{H}, 0,1.61632,1.54340,-1.89146$
2-Butano

$\begin{array}{lllll}\mathrm{H} & 0 & 0.38399 & 2.06379 & -1.04088\end{array}$

$\begin{array}{lllll}\mathrm{C} & 0 & 0.38262 & 2.03049 & 0.04135\end{array}$

$\begin{array}{llllll}\mathrm{H} & 0 & 1.41571 & 2.02896 & 0.379\end{array}$

$\begin{array}{llllll}\text { C } & 0 & -0.36336 & 0.79753 & 0.54812\end{array}$

$\begin{array}{llllll}\mathrm{H} & 0 & -0.08266 & 2.93986 & 0.40917\end{array}$

$\begin{array}{llllll}\text { C } & 0 & 0.24507 & -0.51673 & 0.07446\end{array}$

$\begin{array}{lllll}\mathrm{H} & 0 & -1.398 & 0.83112 & 0.21693\end{array}$

$\begin{array}{lllll}\mathrm{H} & 0 & -0.37796 & 0.79796 & 1.63532\end{array}$

$\begin{array}{lllll}\mathrm{H} & 0 & 1.29182 & -0.54395 & 0.37914\end{array}$

$\begin{array}{llllll}\mathrm{O} & 0 & 0.16839 & -0.52523 & -1.3333\end{array}$

$\begin{array}{llllll}\text { C } & 0 & -0.47042 & -1.72926 & 0.66015\end{array}$

$\begin{array}{lllll}\mathrm{H} & 0 & -0.0304 & -2.65348 & 0.29334\end{array}$

$\begin{array}{lllll}\mathrm{H} & 0 & -1.5181 & -1.72188 & 0.378\end{array}$

$\begin{array}{llllll}\mathrm{H} & 0 & -0.4025 & -1.73944 & 1.74355\end{array}$

H $0 \quad 0.6076 \quad-1.29327 \quad-1.671$

2,2-Dimethyl-1-propanol

C, $0,0.35906,0.18535,0.01402$

C, $0,0.35921,0.18546,1.54139$

$\mathrm{H}, 0,1,3853,0.18482,1.92352$

$\mathrm{H}, 0,-0.145,1.07659,1.93047$

$\mathrm{H}, 0,-0.15745,-0.69566,1.92727$

C, $0,-1.0764,0.1924,-0.49547$

$\mathrm{H}, 0,-1.58446,1.10231,-0.13910$

$\mathrm{H}, 0,-1.07156,0.21898,-1.59648$

C, $0,1.05275,1.44372,-0.50455$

$\mathrm{H}, 0,2.09071,1.47611,-0.15889$

$\mathrm{H}, 0,1.06259,1.46844,-1.59966$

$\mathrm{H}, 0,0.55113,2.34929,-0.14636$

C, $0,1.08015,-1.05614,-0.50710$

$\mathrm{H}, 0,0.57936,-1.96464,-0.16636$

$\mathrm{H}, 0,1.09772,-1.06479,-1.60255$

$\mathrm{H}, 0,2.115,-1.07355,-0.15263$

O,0,-1.74007,-0.97837,-0.01939

$\mathrm{H}, 0,-2.65176,-0.95610,-0.3537$
1-Pentanol

C, $0,-1.63045,-0.20436,-2.51066$ C, $0,-1.57070,-0.18282,-0.97988$ C, $0,-0.13847,-0.21100,-0.43199$ C, $0,-0.07570,-0.1966,1.10020$ C $0,1.34380,-0.19448,1.6551$ $\mathrm{O}, 0,1.98359,1.01190,1.2446$ $\mathrm{H}, 0,-2.66478,-0.17791,-2.87256$ $\mathrm{H}, 0,2.90111,0.98376,1.55432$ $\mathrm{H}, 0,-1.10495,0.65802,-2.93886$ $\mathrm{H}, 0,-1.15987,-1.11006,-2.91309$ $\mathrm{H}, 0,-2.08255,0.71576,-0.60683$ $\mathrm{H}, 0,-2.13198,-1.03971,-0.57962$ $\mathrm{H}, 0,0.37572,-1.10962,-0.80624$ $\mathrm{H}, 0,0.42281,0.64826,-0.8188$ $\mathrm{H}, 0,-0.60106,-1.07310,1.50580$ $\mathrm{H}, 0,-0.59701,0.69013,1.48560$ $\mathrm{H}, 0,1.89053,-1.07723,1.2819$ $\mathrm{H}, 0,1.31253,-0.26731,2.7547$

2-Pentanol

C, $0,0.02476,0 ., 0.21119$

$\mathrm{H}, 0,0.02898,0 ., 1.2963$

$\mathrm{O}, 0,1.3895,0 .,-0.14548$

H,0,1.46349,0.,-1.09112

C, $0,-0.67292,-1.27046,-0.27669$

C, $, 0,-0.67292,1.27046,-0.27669$

C, $0,-0.04878,-2.55286,0.27114$

C, $0,-0.04878,2.55286,0.27114$

$\mathrm{H}, 0,-1.72207,-1.22477,0.00466$

$\mathrm{H}, 0,-1.72207,1.22477,0.00466$

$\mathrm{H}, 0,-0.65054,-1.28730,-1.36654$ $\mathrm{H}, 0,-0.65054,1.28730,-1.36654$ $\mathrm{H}, 0,-0.10995,-2.58174,1.35533$ $\mathrm{H}, 0,-0.10995,2.58174,1.35533$ $\mathrm{H}, 0,-0.56289,-3.42865,-0.11254$ H, $0,-0.56289,3.42865,-0.11254$ $\mathrm{H}, 0,0.99693,-2.62624,-0.00186$ H, $0,0.99693,2.62624,-0.00186$
1,2-Propanediol

$\mathrm{H}, 0,0.7426,1.4930,-0.9520$ C, $0,0.6271,1.5363,0.1390$ $\mathrm{H}, 0,1.6258,1.5847,0.5911$ $\mathrm{C}, 0,-0.1695,0.3328,0.6563$ $\mathrm{H}, 0,0.0944,2.4624,0.3733$ C, $0,0.4821,-1.0117,0.3077$ $\mathrm{O}, 0,-1.4999,0.3457,0.1527$ $\mathrm{H}, 0,-0.2518,0.4033,1.748$ $\mathrm{H}, 0,1.524976546,-1.0546,0.6547$ O, $0,0.3871,-1.2731,-1.0966$ $\mathrm{H}, 0,-0.0756,-1.8197,0.7895$ $\mathrm{H}, 0,-1.4404,-0.0740,-0.7243$ $\mathrm{H}, 0,1.0444,-0.7213,-1.5480$

1,3-Propanediol

C, $0,0.2901,0 ., 0.1954$ $\mathrm{H}, 0,0.290,0 ., 1.2982$ $\mathrm{H}, 0,1.3386,0 .,-0.123$ $\mathrm{C}, 0,-0.3900,-1.2780,-0.3020$ C, $0,-0.3900,1.2780,-0.3020$ $\mathrm{H}, 0,-1.4493,-1.2903,0.0059$ $\mathrm{O}, 0,0.2787,-2.4612,0.1178$ $\mathrm{H}, 0,-0.3742,-1.3101,-1.3969$ $\mathrm{H}, 0,-1.4493,1.2903,0.0059$ $\mathrm{H}, 0,-0.3742,1.3101,-1.3969$ $\mathrm{O}, 0,0.2787,2.4612,0.1178$ $\mathrm{H}, 0,0.2483,2.4854,1.0869$ $\mathrm{H}, 0,0.2483,-2.4854,1.0869$

2-Methyl-2-butanol
C, $0,-1.05902,0 .,-1.888$
C, $0,-1.140,0 .,-0.36165$
C, $0,0.19404,0.0 .3960$
C, $0,1.01664,1.25930,0.11280$
$\mathrm{C}, 0,1.01664,-1.25930,0.11280$
$\mathrm{O}, 0,-0.1955,0 ., 1.75853$
$\mathrm{H}, 0,-2.05906,0 .,-2.31037$
$\mathrm{H}, 0,-0.54827,0.87748,-2.27159$
$\mathrm{H}, 0,-0.54827,-0.87748,-2.2715$
$\mathrm{H}, 0,-1.69967,0.86868,-0.02835$
$\mathrm{H}, 0,-1.69967,-0.86868,-0.0283$
$\mathrm{H}, 0,0.5765,0 ., 2.3081$
$\mathrm{H}, 0,1.3539,1.3025188761,-0.9166$
$\mathrm{H}, 0,0.4316,2.1478594136,0.3243$
$\mathrm{H}, 0,1.9010,1.282362173,0.7453$
$\mathrm{H}, 0,1.3539,-1.3025,-0.9166$
$\mathrm{H}, 0,1.9010,-1.282,0.7453$
$\mathrm{H}, 0,0.4316,-2.1478,0.3243$ 
1-Hexanol

$\mathrm{O}, 0,-1.5826,0 .,-3.3407$

C, $0,-1.74880,0 .,-1.9463$

$\mathrm{C}, 0,-0.37268,0 .,-1.3044$

C, $0,-0.42899,0 ., 0.2239$

C, $0,0.95616,0 ., 0.87318$

C, $0,0.90788,0 ., 2.40210$

C, $0,2.29511,0.3 .04349$

$\mathrm{H}, 0,-2.3089,-0.8778,-1.6270$

$\mathrm{H}, 0,-2,3089,0.8778,-1.62702$

$\mathrm{H}, 0,0.17010,-0.8717668,-1.6593$

$\mathrm{H}, 0,0.17010,0.8717668,-1$.

$\mathrm{H}, 0,-0.98479,0.87116,0.56744$

$\mathrm{H}, 0,-0.98479,-0.87116,0.56744$

$\mathrm{H}, 0,1.51315,-0.87063,0.53139$

$\mathrm{H}, 0,1.51315,0.87063,0.53139$

H, $0,0.35225,0.87044,2.74483$

H, $, 0.35225,0.87044,2.74483$

$\mathrm{H}, 0,0.35225,-0.87044,2.7448$.
$\mathrm{H}, 0,2.22804,0 ., 4.12712$

$\mathrm{H}, 0,2.22804,0 ., 4.12712$
$\mathrm{H}, 0,2.86391,-0.87693,2.7473$

$\mathrm{H}, 0,2.86391,0.87693,2.7473$

$\mathrm{H}, 0,-2.43020,0 .,-3.76225$

1-Heptanol

C, $0,-0.99776,1.42193,-0.37895$

C, $0,-0.39569,0.05289,-0.69991$

C, $0,0.69267,-0.37340,0.28691$

C, $0,1.29113,-1.74184,-0.04237$

C, $0,2.3692,-2.18416,0.93225$

O, $0,1.79156,-2.33758,2.20423$

$\mathrm{H}, 0,2.45897,-2.58080,2.82975$

$\mathrm{H}, 0,-1.40775,1.40654,0.6294$

$\mathrm{H}, 0,-0.20730,2.17092,-0.37395$

$\mathrm{H}, 0,-1.18733,-0.69453,-0.70579$

$\mathrm{H}, 0,0.01488,0.06881,-1.70858$

$\mathrm{H}, 0,1.48653,0.37256,0.29092$

$\mathrm{H}, 0,0.28588,-0.39672,1.29256$

$\mathrm{H}, 0,1.72271,-1.72519,-1.04155$

H, $0,0.50777,-2.49528,-0.04916$

H, $0.0 .1697,-1.4528,-0.04916$

$\mathrm{H}, 0,3.16916,-1.44532,0.96242$

$\mathrm{H}, 0,2.80347,-3.12447,0.59719$

C, $0,-2.09016,1.8497,-1.36073$

$\mathrm{H}, 0,-1.68177,1.86622,-2.36913$

$\mathrm{H}, 0,-2.88203,1.10369,-1.36530$

C, $0,-2.6861,3.21859,-1.03346$

$\mathrm{H}, 0,-1.92447,3.99310,-1.05484$

$\mathrm{H}, 0,-1.92447,3.99310,-1.05484$

H, $0,-3.13383,3.22461,-0.04363$
1-Octanol

C, $0,-0.55579,1.03459,-0.13272$ C, $0,0.0132,-0.34533,-0.46762$ C, $0,1.11121,-0.79501,0.4979$ C, $0,1.67653,-2.17413,0.15498$ C, $0,2.76378,-2.63960,1.10841$ $\mathrm{O}, 0,2.20717,-2.784,2.39074$

$\mathrm{H}, 0,2.88131,-3.04213,3.00314$

$\mathrm{H}, 0,-0.9466540359,1.02415,0.8832$ $\mathrm{H}, 0,0.24966,1.76725,-0.140895821$

$\mathrm{H}, 0,-0.79273,-1.07716,-0.46023$

$\mathrm{H}, 0,0.40535,-0.335141 .48369$

$\mathrm{H}, 0,1.91949,-0.06473,0.48868$

$\mathrm{H}, 0,0.72279,-0.8126,1.51100$

$\mathrm{H}, 0,2.08965,-2.16365,-0.8520$

$\mathrm{H}, 0,0.87859,-2.91212,0.16139$

$\mathrm{H}, 0,3.57850,-1.916636172,1.12491$

$\mathrm{H}, 0,3.17313,-3.58750,0.76353$

C, $0,-1.6575,1.48503,-1.09363$

$\mathrm{H}, 0,-1.26733,1.49504,-2.11024$

$\mathrm{H}, 0,-2.46389,0.75338,-1.08532$

C, $0,-2.2269,2.86509,-0.75977$

$\mathrm{H}, 0,-1.42286,3.59805,-0.7691$

$\mathrm{H}, 0,-2.61899,2.85658,0.25505$

C, $0,-3.32700,3.30791,-1.72397$

$\mathrm{H}, 0,-3.71017,4.28925,-1.46132$

H, $0,-2.95677,3.35979,-2.74408$

H,0,-4.16198,2.61295,-1.71186
1-Nonanol

C, $0,-0.24815,0.47196,0.23792$

C, $0,0.28753,-0.92821,-0.06702$

C, $0,-0.32004,-1.54469,-1.32835$

$\mathrm{C}, 0,0.21835,-2.94525,-1.62474$

C, $0,-0.36910,-3.56955,-2.87899$

$\mathrm{O}, 0,0.00917,-2.7941,-3.98839$

$\mathrm{H}, 0,-0.37833,-3.15216,-4.7742$

$\mathrm{H}, 0,-0.05641,1.12229,-0.61370$

$\mathrm{H}, 0,-1.33045,0.42662,0.34790$

$\mathrm{H}, 0,1.36982,-0.88235,-0.17582$

$\mathrm{H}, 0,0.09499,-1.57964,0.7843$

$\mathrm{H}, 0,-1.40278,-1.59361,-1.21892$

$\mathrm{H}, 0,-0.12464,-0.90186,-2.18036$

$\mathrm{H}, 0,0.0084,-3.60645,-0.78594$

$\mathrm{H}, 0,1.29895,-2.90933,-1.73589$

$\mathrm{H}, 0,-1.45442,-3.61644,-2.79776$

$\mathrm{H}, 0,-0.00388,-4.58935,-2.9870$

$\mathrm{C}, 0,0.36151,1.09336,1.49600$

$\mathrm{H}, 0,0.17037,0.44225,2.34756$

$\mathrm{H}, 0,1.44371,1.13914,1.38573$

$\mathrm{C}, 0,-0.17446,2.49344,1.80084$

H, $0,-1.2567,2.44863,1.91180$

$\mathrm{H}, 0,0.01648,3.14531,0.94983$

C, $0,0.43477,3.11611,3.05827$

$\mathrm{H}, 0,0.24345,2.46705,3.91031$

$\mathrm{H}, 0,1.51619,3.16362,2.94859$

C, $0,-0.105720082,4.51430,3.35565$

$\mathrm{H}, 0,0.34494,4.9289,4.25222$

$\mathrm{H}, 0,0.10056,5.19691,2.53598$

H, $0,-1.18169,4.49519,3.50507$
1-Decanol

C, $0,-0.30395,0.01435,-0.18304$ C, $0,0.19980,-1.39685,-0.49165$ C, $0,-0.43896,-2.00534,-1.74142$ C, $0,0.06768,-3.41692,-2.04147$ C, $, 0,-0.55108,-4.03361,-3.28440$ $\mathrm{O}, 0,-0.17524,-3.2690,-4.40212$ $\mathrm{H}, 0,-0.58172,-3.62192,-5.18064$ $\mathrm{H}, 0,-0.11293,0.6582,-1.0396$ $\mathrm{H}, 0,-1.38511,-0.00972,-0.05627$ $\mathrm{H}, 0,1.2809,-1.37226,-0.61728$ H, $0,0.0079,-2.04189,0.36468$ $\mathrm{H}, 0,-1.52062,-2.03302,-1.61515$ $\mathrm{H}, 0,-0.24444,-1.36897,-2.59847$ $\mathrm{H}, 0,-0.14184,-4.07141,-1.19733$ H, $0,1.14692,-3.40224,-2.16944$ $\mathrm{H}, 0,-1.63570,-4.05929,-3.18623$ H, $0,-0.2072,-5.06059,-3.39472$ $\mathrm{C}, 0,0.33691,0.62775,1.06343$ $\mathrm{H}, 0,0.14646,-0.01695,1.91995$ $\mathrm{H}, 0,1.41793,0.65221,0.93636$ C, $0,-0.16719,2.03903,1.37188$ $\mathrm{H}, 0,-1.24829,2.01465,1.49907$ $\mathrm{H}, 0,0.02313,2.68361,0.51533$ C, $0,0.4738832238,2.65282,2.6179$ $\mathrm{H}, 0,0.28366,2.00907,3.47531$ $\mathrm{H}, 0,1.555105216,2.67811,2.49145$ C, $0,-0.0294946915,4.0639,2.92691$ $\mathrm{H}, 0,0.16109,4.70937,2.07205$ $\mathrm{H}, 0,-1.10963,4.04075,3.05558$ C, $0,0.61568,4.66996,4.17250$ $\mathrm{H}, 0,1.6941,4.73910,4.06112$ $\mathrm{H}, 0,0.23872,5.66981,4.36523$ $\mathrm{H}, 0,0.41374,4.06529,5.05226$ 
Cyclopropanol

C, $0,-0.259,-0.43,-1.0705$

C, $0,-0.2256,-0.2579,0.4141$

C, $0,1.0721,-0.3738,-0.3455$

$\mathrm{H}, 0,-0.5958,-1.3813,-1.4772$

$\mathrm{H}, 0,-0.5476,0.4405,-1.6490$

$\mathrm{H}, 0,-0.5305,-1.1051,1.0318$

$\mathrm{H}, 0,1.6572,0.5345,-0.460$

$\mathrm{H}, 0,1.6576,-1.2844,-0.247$

$0,0,-0.6336,0.997,0.8924$

H, $0,-0.0958,1.1990,1.6746$

Cyclohexano

C, $0,0.7500,-1.7254,0$

$\mathrm{H}, 0,0.7009,-2.8107,0$.

$\mathrm{H}, 0,1.8064,-1.4613,0$.

C, $0,0.0892,-1.1676,1.2642$

C, $0,0.0892,-1.1676,-1.2642$

$\mathrm{H}, 0,0.6018,-1.5319,2.1497$

$\mathrm{H}, 0,-0.9337,-1.5351,1.3264$

$\mathrm{H}, 0,0.6018,-1.5319,-2.1497$

$\mathrm{H}, 0,-0.9337,-1.5351,-1.3264$

C, $0,0.076,0.3644,1.2605$

C, $0,0.076,0.3644,-1.2605$

$\mathrm{H}, 0,-0.4404,0.749,2.1339$

$\mathrm{H}, 0,1.1012,0.7345,1.3113$

$\mathrm{H}, 0,-0.4404,0.749,-2.1339$

$\mathrm{H}, 0,1.1012,0.7345,-1.3113$

C, $0,-0.583,0.9208,0$.

$\mathrm{O}, 0,-0.6010,2.3273,0$.

$\mathrm{H}, 0,-1.6301,0.6336,0$.

$\mathrm{H}, 0,0.2901,2.6504,0$.

Cyclopentanol

$\mathrm{H}, 0,0.1390,0.1428,-0.0175$

C, $0,0.0704,0.0388,1.0591$

C, $0,1.4364,0.0309,1.7355$

C, $0,-0.6954,1.1927,1.7368$

$\mathrm{H}, 0,-0.4035,-0.9160,1.2643$

C $, 0,1.1009,0.3539,3.1916$

C, $0,0.0370,1.4568,3.08$

C, $0,0.0370,1.4568,3.08$

$\mathrm{H}, 0,2.051,0.8310,1.3215$
$\mathrm{O}, 0,2.0660,-1.2058,1.5373$

$\mathrm{H}, 0,-0.6834,2.0833,1.117$

$\mathrm{H}, 0,-1.7373,0.9358,1.8897$

$\mathrm{H}, 0,0.6872,-0.5415,3.6475$

$\mathrm{H}, 0,1.9653,0.653,3.7777$

$4,0,0.5105,2.4335,3.0665$

H, $0,-0.6400,1.4532,3.9295$

$\mathrm{H}, 0,2.9266,-1.1901,1.9321$
Cyclobutanol

C, $0,-0.4507,-0.0298,-1.0775$

$\mathrm{C}, 0,-0.4481,-0.2230,0.4501$

C, $0,1.0831,-0.0602,0.4342$

C, $0,1.0455,-0.4158,-1.0691$

$\mathrm{H}, 0,-1.1397,-0.6223,-1.6679$

$\mathrm{H}, 0,-0.5847,1.0178,-1.3345$

$\mathrm{H}, 0,-0.7210,-1.2383,0.7065$

$\mathrm{H}, 0,1.6711,-0.6780,1.102$

$1,0,1.6711,0.6780,1.102$

H, $0,1.1906,-1.4771,-1.237$

$\mathrm{H}, 0,1.1906,-1.4771,-1.237$
$\mathrm{H}, 0,1.7067,0.1314,-1.7290$

$\mathrm{O}, 0,-1.229,0.5948,1.2591$

$\mathrm{H}, 0,-1.0314,1.5030,1.0758$
Ethers

Dipropyl ether

O, $0,-0.081945,0.045514,0.113174$

C, $0,-0.278590,1.397612,0.401994$

C, $0,-0.718703,-0.839203,0.986153$

C, $0,-0.396483,-2.266290,0.574483$

$\mathrm{H}, 0,0.101710,1.624046,1.397886$

$\mathrm{H}, 0,-1.344656,1.62840,0.401619$

$\mathrm{H}, 0,-0.387060,-0.657891,2.009325$

H,0,-1.795896,-0.675687,0.960807

C, $0,1.09108,-2.600723,0.670648$

H, $0,-0.744424,-2.417338,-0.4432$

$\mathrm{H}, 0,-0.969458,-2.939501,1.208115$

$\mathrm{H}, 0,1.277232,-3.627627,0.371769$

$\mathrm{H}, 0,1.4527798,-2.481813,1.68860$

$\mathrm{H}, 0,1.675026,-1.950929,0.030356$

C $, 0,0.442031,2.240694,-0.637188$

$\mathrm{H}, 0,0.352880,3.285300,-0.347541$

$\mathrm{H}, 0,1.499203,1.993427,-0.607482$

C, $0,-0.098151,2.045118,-2.052739$

$\mathrm{H}, 0,0.440197,2.666256,-2.761923$

$\mathrm{H}, 0,0.000667,1.012440,-2.364301$

$\mathrm{H}, 0,-1.149783,2.31355,-2.109523$

Diisopropyl ether

O, $0,-0.003403,0.01,0.48$

C, $0,-1.191736,-0.161673,-0.24$

C, $0,1.195117,0.144867,-0.240321$

$\mathrm{H}, 0,-0.97,-0.719424,-1.156261$

H, $0,0.994960,0.638545,-1.189266$

C, $0,2.125532,1.025008,0.580444$

C, $0,1.799661,-1.228964,-0.514735$

$\mathrm{H}, 0,2.31305,0.570564,1.547662$

$\mathrm{H}, 0,1.676238,1.997678,0.745981$

$\mathrm{H}, 0,3.076306,1.163422,0.074876$

$\mathrm{H}, 0,2.017593,-1.732974,0.421181$

$\mathrm{H}, 0,2.721318,-1.141192,-1.08237$

$\mathrm{H}, 0,1.117369,-1.853648,-1.082036$

C $0,-1.791601,1.189774,-0.62313$

$\mathrm{H}, 0,-1.10114,1.774205,-1.222547$

H, $0,-2.705287,1.062696,-1.196192$

$\mathrm{H}, 0,-2.022325,1.756953,0.272711$

C, $0,-2.133997,-0.983578,0.619960$

$\mathrm{H}, 0,-3.077591,-1.157094,0.111774$

$\mathrm{H}, 0,-1.687476,-1.942246,0.858555$

$\mathrm{H}, 0,-2.334986,-0.463576,1.550766$
Dibutyl ether

$\mathrm{O}, 0,0.253806,0.154835,0.324529$

C $, 0,0.246176,0.162967,1.721246$

C, $0,1.523603,0.213841,-0.254424$

C, $0,1.384933,0.218936,-1.767729$

$\mathrm{H}, 0,0.717680,1.073317,2.091031$

$\mathrm{H}, 0,0.828712,-0.67470,2.101997$

$\mathrm{H}, 0,2.045188,1.111152,0.080578$

$\mathrm{H}, 0,2.117079,-0.643030,0$

C, $0,0.651561,1.445906,-2.311663$

$\mathrm{H}, 0,0.8623,-0.685510,-2.068395$

$\mathrm{H}, 0,2.382477,0.165471,-2.200244$

$\mathrm{H}, 0,1.181378,2.345424,-2.003471$

$\mathrm{H}, 0,-0.334049,1.498639,-1.862420$

C,0,-1.1912,0.09672,2.214506

$\mathrm{H}, 0,-1.176541,0.138531,3.302302$

$\mathrm{H}, 0,-1.705376,0.9906,1.872703$

C. $0,-1.97,-1.136588,1.753941$

$\mathrm{H}, 0,-3.003368,-1.028199,2.092008$

$\mathrm{H}, 0,-2.006538,-1.1516,0.670010$

C, $0,-1.42,-2.46682,2.2688626119$

$\mathrm{H}, 0,-2.069330,-3.289220,1.976195$

$\mathrm{H}, 0,-0.435803,-2.674206,1.871065$

$\mathrm{H}, 0,-1.354407,-2.472828,3.353762$

C. $0,0.525123,1.429180,-3.834542$

$\mathrm{H}, 0,0.002835,2.310161,-4.194762$

$\mathrm{H}, 0,1.501142,1.405540,-4.31182$

$\mathrm{H}, 0,-0.027716,0.55694,-4.172050$ 
Di-sec-butyl ether

$\mathrm{O}, 0,0.260688,-0.306876,0.54157$ C, $0,-1.063232,-0.492343,0.09554$ C, $0,1.28662,-0.330155,-0.421330$ $\mathrm{H}, 0,-1.060650,-1.08911,-0.814879$ C, $0,2.449198,0.495167,0.12293$ C, $0,1.707555,-1.767513,-0.71671$ $\mathrm{H}, 0,0.932190,0.132050,-1.342092$ $\mathrm{H}, 0,2.770404,0.049451,1.06052$ H, $0,3.284219,0.410847,0.568586$ $\mathrm{H}, 0,3.284219,0.410847,0.568586$ $\mathrm{H}, 0,2.084121,-2.238503,0.185432$ $\mathrm{H}, 0,2.487115,-1.795894,-1.472057$ $\mathrm{H}, 0,0.872833,-2.357983,-1.079726$ C,0,2.110167, 1.967782,0.34218 $\mathrm{H}, 0,1.301160,2.076751,1.053698$ $\mathrm{H}, 0,1.808829,2.444575,-0.587080$ $\mathrm{H}, 0,2.971117,2.508201,0.723625$ C, $0,-1.698884,0.866,-0.211742$

$\mathrm{H}, 0,-1.037779,1.405524,-0.883959$ $\mathrm{H}, 0,-1.736652,1.442538,0.708693$ C, $0,-1.789353,-1.268966,1.185155$ $\mathrm{H}, 0,-2.813594,-1.489672,0.90575$ $\mathrm{H}, 0,-1.277788,-2.205884,1.37389$ H, $0,-1.798188,-0.698726,2.108496$ C, $0,-3.090216,0.787065,-0.842287$ $\mathrm{H}, 0,-3.821594,0.357726,-0.166033$ $\mathrm{H}, 0,-3.442023,1.777001,-1.114208$ $\mathrm{H}, 0,-3.080398,0.183889,-1.746539$
Isopropyl tert-butyl ether

$\mathrm{O}, 0,0.590510,0.122847,-0.119278$

C, $0,-0.550169,0.109422,-0.958541$

C, $0,0.494632,-0.180622,1.254406$

$\mathrm{H}, 0,-0.531469,-0.407206,1.522826$

C, $0,1.356102,-1.404602,1.543164$

C, $0,0.951146,1.034379,2.053721$

$\mathrm{H}, 0,2.384847,-1.210692,1.257869$

$\mathrm{H}, 0,1.007336,-2262934,0.979916$ H, $0,1.333778,-1.656724,2.599376$

$\mathrm{H}, 0,1.968724,1.29635,1.78311$

$\mathrm{H}, 0,0.91988,0.83340,3.12062$

$\mathrm{H}, 0,0.31807,1.89048,1.84895$

C, $0,-1.1769,-1.28750,-1.02021$

$\mathrm{H}, 0,-1.58062,-1.59648,-0.06180$

$\mathrm{H}, 0,-1.99151,-1.30556,-1.73729$

H, $0,-0.43661,-2.01765,-1.32836$

C, $0,0.00236,0.48813,-2.33117$

$\mathrm{H}, 0,-0.78659,0.51361,-3.07618$

$\mathrm{H}, 0,0.47058,1.46498,-2.28892$

$\mathrm{H}, 0,0.75100,-0.23089,-2.64385$

$\mathrm{C}, 0,-1.58103,1.15070,-0.51010$

$\mathrm{H}, 0,-1.99767,0.91919,0.46443$

$\mathrm{H}, 0,-1.12444,2.13316,-0.45968$

$\mathrm{H}, 0,-2.40570,1.19464,-1.21436$
Peroxides

Ethyl hydroperoxide

C, $0,1.41547,-0.56948,-0.39665$ $\mathrm{H}, 0,1.28761,-0.77955,-1.45276$ , $0,1.11988,-1.44555,0.1666$ $\mathrm{H}, 0,2.46,-0.37900,-0.20784$ C, $0,0.58889,0.63862,0.00579$ $\mathrm{H}, 0,0.71054,0.86745,1.05946$ $\mathrm{H}, 0,0.87220,1,5115,-0.57020$ $0,0,0.77988,0.4816,-0.28059$ $0,0,-0.77988,0.4816,-0.28059$ O, $, 0,-1.29031,-0.50364,0.56485$

Propyl hydroperoxide

C, $0,0.01021,0.0134,0.00576$

$\mathrm{H}, 0,-0.11388,0.00780,-1.07305$

$\mathrm{H}, 0,-0.29249,-0.96359,0.36493$

C, $0,-0.91536,1.06838,0.59186$

$\mathrm{H}, 0,-0.84012,1.10037,1.67462$

$\mathrm{H}, 0,-0.6732,2.05120,0.20278$

$\mathrm{O}, 0,-2.26046,0.88492,0.22398$

$\mathrm{O}, 0,-2.73541,-0.25938,0.8652$

$\mathrm{H}, 0,-3.41750,0.09773,1.42068$

C, $0,1.47199,0.27590,0.36822$

Н $0,2.118776,-0.48209,-0.060069$ $\mathrm{H}, 0,1.806722,1.240992,-0.002192$ $\mathrm{H}, 0,1.62139,0.264795,1.444321$

Butyl hydroperoxide

C, $0,-0.008195,-0.011893,0.000578$ $\mathrm{H}, 0,0.060590,-0.066195,-1.08270$ $\mathrm{H}, 0,-0.195752,-1.01946,0.356458$ C, $0,-1.190576,0.872849,0.36445$ , $, 0,-1.057676,0.872849,0.364451$ H, $0,-1.059810,1.873500,-0.032225$ $\mathrm{H}, 0,-1.059810,1.873500,-0.032225$
$\mathrm{O}, 0,-2.39804,0.440134,-0.213624$ $\mathrm{O}, 0,-2.772585,-0.754444,0.401903$ $\mathrm{H}, 0,-3.592449,-0.511882,0.814632$ C, $0,1.305650,0.511511,0.584738$ $\mathrm{H}, 0,1.483715,1.525883,0.23287$ $\mathrm{H}, 0,1.222476,0.574302,1.667939$ C, $0,2.503391,-0.365410,0.221516$ $\mathrm{H}, 0,2.635693,-0.420270,-0.855039$ H,0,3.420888,0.026714,0.648907 H,0,2.37191,-1.378536,0.589934
tert-Butyl hydroperoxide

C, $0,-0.02845,-0.40064,0.00541$ C, $0,-0.01070,-0.39883,1.53286$ $\mathrm{H}, 0,1.01278,-0.40373,1.88917$ $\mathrm{H}, 0,-0.51699,-1.27840,1.91173$ $\mathrm{H}, 0,-0.50490,0.48568,1.90760$ C, $0,0.76303,-1.57956,-0.56674$ $\mathrm{H}, 0,1.78114,-1.55858,-0.19870$ $\mathrm{H}, 0,0.29928,-2.51276,-0.27091$ H, $0,0.78390,-1.52108,-1.64742$ C, $0,-1.44967,-0.35617,-0.55294$ $\mathrm{C}, 0,-1.44967,-0.35617,-0.5529$ $\mathrm{H}, 0,-1.41673,-0.32239,-1.63490$ $\mathrm{H}, 0,-1.99641,-1.2389,-0.24313$ $\mathrm{H}, 0,-1.96350,0.5253,-0.19755$ $\mathrm{O}, 0,0.72863,0.75328,-0.4933$ $0,0,0.03195,1.95768,-0.02296$ $\mathrm{H}, 0,0.79153,2.54840,0.1027$

Dimethyl peroxide

O, $0,0.0165,0.1836,-0.67454$ $\mathrm{O}, 0,-0.0168,-0.1838,0.67448$ C, $0,0.8093,-0.7720,-1.31377$ $\mathrm{H}, 0,1.8144,-0.7708,-0.9091$ $\mathrm{H}, 0,0.3758,-1.7600,-1.2142$ $\mathrm{H}, 0,0.8308,-0.4820,-2.3555$ C, $0,-0.8091,0.77,1.3138$

$\mathrm{H}, 0,-0.3754,1.7600,1.2141$ $\mathrm{H}, 0,-1.8144,0.7711,0.9094$ $\mathrm{H}, 0,-0.8305,0.4821,2.3556$

Diethyl peroxide

$\mathrm{O}, 0,-0.0122,0.0831,-0.705$ $\mathrm{O}, 0,-0.2032,-0.22993,0.640535$ C, $0,1.23007,-0.4469,-1.0960$ $\mathrm{H}, 0,2.0041,-0.072,-0.4347$

$\mathrm{H}, 0,1.3908,-0.0294,-2.0829$ C, $0,-0.4617,0.9698,1.3267$ $\mathrm{H}, 0,0.3447,1.6709,1.1400$ $\mathrm{H}, 0,-0.4312,0.6825,2.371$ C, $0,1.232,-1.96,-1.144$ $\mathrm{H}, 0,0.4567,-2.3228,-1.8119$ $\mathrm{H}, 0,2.1916,-2.323,-1.5062$ $\mathrm{H}, 0,1.0595,-2.3834,-0.1607$ C $, 0,-1.8096,1.5719,0.9707$ $\mathrm{H}, 0,-1.9882,2.4618,1.5673$ $\mathrm{H}, 0,-1.8462,1.848,-0.0754$ $\mathrm{H}, 0,-2.6046,0.8609,1.1669$ 


\section{Aldehydes/Ketones}

Pentanal

C, $0,-2.2085,0 ., 0.21727$

$\mathrm{O}, 0,-2.1934,0 ., 1.40550$

C, $0,-0.9839,0 .,-0.66187$

C, $0,0.3350,0 ., 0.10458$

C, $0,1.5533,0 .,-0.82023$

C, $0,2.8751,0 .,-0.05386$

$\mathrm{H}, 0,-3.1635,0,-0.32075$

$\mathrm{H}, 0,-1.0577,-0.86648,-1.3190$

$\mathrm{H}, 0,-1.0577,0 .,-1.3190$

$\mathrm{H}, 0,0.3670,0.8675,0.7566$

$\mathrm{H}, 0,0.3670,-0.8675,0.756$

$\mathrm{H}, 0,1.5146,-0.8705,-1.4719$

$\mathrm{H}, 0,1.5146,0.8705,-1.4719$

$\mathrm{H}, 0,3.7221,0,-0.732$

$\mathrm{H}, 0,2.9589,0.8769,0.5814$

H, $0,2.9589501278,-0.8769,0.5814$

2-Methylbutanal

$\mathrm{H}, 0,0.3450,2.3628,-1.4167$ C, $0,0.357,2.3559,-0.3325$

$\mathrm{H}, 0,1.3946,2.3619,-0.0096$

C, $0,-0.3900,1.1531,0.2437$

$\mathrm{H}, 0,-0.0984,3.2809,0.0042$

C, $0,0.2541,-0.2069,-0.0859$

$\mathrm{H}, 0,-1.4184,1.1613,-0.1127$

$\mathrm{H}, 0,-0.4382,1.2584,1.3245$

$\mathrm{H}, 0,1.2737,-0.2203,0.285$

C, $, 0,0.2472,-0.5321,-1.586$

C, $0,-0.4885,-1.2922,0.6585$

$\mathrm{H}, 0,0.8323,0.1872,-2.1457$

$\mathrm{H}, 0,0.8323,0.1872,-2.1457$

$\mathrm{H}, 0,-0.7633,-0.5224,-1.9862$

$\mathrm{H}, 0,0.6718,-1.5136,-1.772$

$\mathrm{O}, 0,-0.0183,-1.9747,1.5102$

$\mathrm{H}, 0,-1.5371,-1.4252,0.3616$
3-Methylbutanal

C, $0,0.0222,0.4717,-0.827$

C, $0,0.0487,0.4657,0.7189$

$\mathrm{H}, 0,1.0660,0.4893,-1.1779$

C, $0,-0.6447,-0.7973,-1.3785$

C, $0,-0.6702,1.7371,-1.3517$

$\mathrm{H}, 0,-0.9606,0.3609,1.1330$

C, $0,0.9193,-0.6352,1.2796$

$\mathrm{H}, 0,0.4647,1.42,1.0716$

$\mathrm{H}, 0,-1.7135,1.7812,-1.0147$

$\mathrm{H}, 0,-0.6739,1.7558,-2.4477$

$\mathrm{H}, 0,-0.1663,2.64,-1.0008$

$\mathrm{H}, 0,-1.6941,-0.8537,-1.0626$

$\mathrm{H}, 0,-0.145,-1.7073,-1.0263$

$\mathrm{H}, 0,-0.6242,-0.8057,-2.4743$

$\mathrm{O}, 0,0.5526,-1.4904,2.054$

$\mathrm{H}, 0,1.9748,-0.6170,0.9167$

Cyclohexanone

C, $0,-0.0075,0 ., 1.1443$

C, $0,0.2219,0 .,-1.7631$

C, $0,-0.2105,1.2580,-1.0140$

C, $0,0.3522,1.2756,0.413$

C, $0,-0.2105,-1.2580,-1.0140$

C, $0,0.3522,-1.2756,0.413$

$\mathrm{H}, 0,1.3144,0 .,-1.8770$

, $0,-0.200,0 .,-2.7742$

H, $0,-1.3063,1.2927,-0.9668$

$\mathrm{H}, 0,0.1099,2.1584,-1.5495$

$\mathrm{H}, 0,1.4478,1.3471,0.3665$

$\mathrm{H}, 0,-0.0189,2.1245,0.9940$

$\mathrm{H}, 0,-1.3063,-1.2927,-0.9668$

H, $0,0.1099,-2.1584,-1.5495$

$\mathrm{H}, 0,1.4478831129,-1.3471,0.3665$

$\mathrm{H}, 0,-0.0189,-2.1245,0.9940$

$\mathrm{O}, 0,-0.5708,0 ., 2.2350$
Heptanal

C, $0,-3.451095,-0.000888,0.248423$

$\mathrm{O}, 0,-3.47851,-0.000132,1.436391$

$\mathrm{C}, 0,-2.196064,-0.00099,-0.586512$

$\mathrm{C}, 0,-0.904768,-0.00014,0.225964$

C, $0,0.34462,-0.000289,-0.656165$

C, $0,1.64460,0.000573,0.14973$

$\mathrm{H}, 0,-4.386503,-0.00,-0.323046$

$\mathrm{H}, 0,-2.24634,-0.867818,-1.245350$

$\mathrm{H}, 0,-2.246857,0.865047,-1.246342$

$\mathrm{H}, 0,-0.896406,0.867711,0.878168$

$\mathrm{H}, 0,-0.895898,-0.867257,0.879162$

$\mathrm{H}, 0,0.327767,-0.871536,-1.309232$

$\mathrm{H}, 0,0.327247,0.870184,-1.310248$

$\mathrm{H}, 0,1.6622,0.871511,0.80254$

$\mathrm{H}, 0,1.662798,-0.869574,0.803583$

C, $0,2.898858,0.00042,-0.725875$

$\mathrm{H}, 0,2.882731,-0.870390,-1.378006$

$\mathrm{H}, 0,2.882231,0.870471,-1.379018$

C,0,4.193557,0.001266,0.086109

$\mathrm{H}, 0,4.256409,-0.875257,0.724783$

$\mathrm{H}, 0,5.064507,0.001159,-0.561979$

$\mathrm{H}, 0,4,255887,0.878549,0.723791$

Octanal

$\mathrm{C}, 0,4.04452,-0.352752,-0.493450$

$\mathrm{O}, 0,4.071372,-1.54074,-0.496713$ C, $0,2.798947,0.482571,-0.34153$ C, $0,1.516736,-0.329321,-0.1850$ C, $0,0.276801,0.553283,-0.033793$ C, $0,-1.01410,-0.252139,0.123754$ $\mathrm{H}, 0,4.973239,0.218464,-0.606922$ $4,0,4.973239,0.218464,-0.606922$

,

H,0,2.744406,1.14184,-1.207788 H, $0,1.402871,-0.982112,-1.044993$ H, $0,1.613012,-0.981975,0.677206$ $\mathrm{H}, 0,0.399565,1.206914,0.828478$ $\mathrm{H}, 0,0.188633,1.206803,-0.900370$ $\mathrm{H}, 0,-1.136743,-0.905348,-0.73826$ , $0,-0.925856,-0.905302,0.990004$ , $0,-0.925856,-0.905302,0.990004$ C, $0,-2.258227,0.624709,0.275540$ $\mathrm{H}, 0,-2.135648,1.278288,1.137821$ $\mathrm{H}, 0,-2.346547,1.278305,-0.590908$ C, $0,-3.550030,-0.179387,0.433122$ $\mathrm{H}, 0,-3.46420,-0.831927,1.299363$ $\mathrm{H}, 0,-3.674978,-0.83190,-0.428363$ $C, 0,-4.788646,0.703047,0.584239$ H, $0,-4.921912,1.343753,-0.282966$ $\mathrm{H}, 0,-4.921912,1.343753,-0.282966$
$\mathrm{H}, 0,-5.687630,0.104398,0.69390$ H, $0,-4.709515,1.343729,1.458068$
Nonanal

C, $0,-4.692444,0.312876,0.344714$ $\mathrm{O}, 0,-4.723989,1.500761,0.344833$ $\mathrm{C}, 0,-3.437841,-0.517802,0.25391$ $\mathrm{C}, 0,-2.152670,0.29880,0.157875$ C, $0,-0.903592,-0.5792,0.067567$ C, $0,0.390336,0.230902,-0.029132$ $\mathrm{H}, 0,-5.623355,-0.261808,0.414188$ H, $0,-3.422416,-1.175764,1.122739$ H, $0,-3.549565,-1.17889,-0.605438$ H, $0,-2.209949,0.949987,-0.708924$ $\mathrm{H}, 0,-2.082642,0.953125,1.021369$ $\mathrm{H}, 0,-0.854635,-1.231293,0.938350$ $\mathrm{H}, 0,-0.982452,-1.234469,-0.79862$ $\mathrm{H}, 0,0.341250,0.882521,-0.899611$ $\mathrm{H}, 0,0.468991,0.88576,0.836730$ C, $0,1.643642,-0.641589,-0.119706$ H $0,1.69187,-1.29339,0.751107$ $\mathrm{H}, 0,1.564051,-1.296720,-0.985712$ C, $0,2.937907,0.167980,-0.216517$ $\mathrm{H}, 0,3.018392,0.823251,0.64928$ $\mathrm{H}, 0,2.89058,0.819915,-1.087268$ C, $0,4.192269,-0.702958,-0.307162$ H $0,4.114277,-1.357362,-1.172867$ $\mathrm{H}, 0,4.242049,-1.35401,0.563135$ C, $0,5.480917,0.113036,-0.403581$ $\mathrm{H}, 0,5.605550,0.753171,0.465281$ $\mathrm{H}, 0,6.351951,-0.531977,-0.46644$ $\mathrm{H}, 0,5.476822,0.749791,-1.28378$ 
2-Nonanone

C, $0,-0.060218,-0.386283,-0.420864$ $\mathrm{H}, 0,0.614386,-0.404373,-1.275625$ $\mathrm{H}, 0,-0.306591,-1.42527,-0.206755$ C, $0,0.657218,0.226872,0.7829$ $\mathrm{H}, 0,0.908121,1.262289,0.575269$ $\mathrm{H}, 0,-0.009003,0.245677,1.639655$ C, $0,1.928437,-0.53085,1.154549$ $\mathrm{H}, 0,1.704208,-1.573338,1.376771$ C, $0,3.939327,-0.663074,2.762061$ H $, 0,4.375943,-0.162381,3.615000$ $\mathrm{H}, 0,4.375943,-0.162381,3.615000$
$\mathrm{H}, 0,3.727480,-1.697120,3.018046$ C, $0,2.668556,0.049438,2.346414$ $\mathrm{O}, 0,2.277863,1.013623,2.929754$ $\mathrm{H}, 0,2.620231,-0.557891,0.313641$ $\mathrm{H}, 0,4.652995,-0.673645,1.943396$ C, $0,-1.336639,0.365583,-0.802554$ $\mathrm{H}, 0,-1.09058,1.403987,-1.017029$ $\mathrm{H}, 0,-2.011262,0.383424,0.05148$ C, $0,-2.05936,-0.242291,-2.005869$ $\mathrm{H}, 0,-2.305616,-1.28114,-1.791519$ $\mathrm{H}, 0,-1.384632,-0.26023,-2.860399$ C, $0,-3.335980,0.508818,-2.388446$ $\mathrm{H}, 0,-3.091687,1.546693,-2.604604$ H, $0,-4.01214,0.526369,-1.536340$ C,0,-4.05251,-0.104064,-3.591215 $\mathrm{H}, 0,-4.341172,-1.132631,-3.39345$ $\mathrm{H}, 0,-4.952928,0.450169,-3.83768$ H, $0,-3.413619,-0.104432,-4.46996$

\section{Acetal/Ketal}

Dimethoxymethane

C, $0,-0.91945,-0.92898,-1.40218$

$\mathrm{O}, 0,-0.92927,-0.74231,0.00666$

$\mathrm{C}, 0,0.35520,-0.64217,0.56297$

$\mathrm{O}, 0,1.05413,0.51657,0.19123$

C, $0,0.48812,1.70878,0.71854$

$\mathrm{H}, 0,-1.96160,-1.02989,-1.71439$

H, $0,-0.46263,-0.07836,-1.922$

H, $0,-0.37131,-1.84378,-1.67647$

$\mathrm{H}, 0,0.20044,-0.67763,1.65081$

$\mathrm{H}, 0,0.99179,-1.47779,0.23879$

$\mathrm{H}, 0,1.13072,2.53203,0.39750$

$\mathrm{H}, 0,-0.53081,1.87441,0.34796$

$\mathrm{H}, 0,0.46126,1.68116,1.81903$

\section{Carboxylic acids}

Propanoic acid
C $0,0.35820,-0.00005,0.75975$ , $, 0,0.35820,-0.00005,0.75975$ $\mathrm{O}, 0,0.23409,-0.00015,1.97187$
$\mathrm{O}, 0,1.58027,0.0000,0.15549$ $\mathrm{H}, 0,2.23097,-0.00004,0.8877$ $\mathrm{C}, 0,-0.75967,0.00001,-0.25066$

$\mathrm{H}, 0,-1.67960,-0.0000,0.3417$

C $0,-0.70623,1.2638,-1.1103$

C, $0,-0.70623,1.2638,-1.1103$

$\mathrm{H}, 0,0.21074,1.2848,-1.703$

$\mathrm{H}, 0,-1.56130,1.2855,-1.7917$

C, $0,-0.70620,-1.2636,-1.1105$

$\mathrm{H}, 0,0.21078,-1.2845,-1.7034$

$\mathrm{H}, 0,-0.7408,-2.1659,-0.4935$

$\mathrm{H}, 0,-1.5612,-1$

2-Methylpropanoic acid

C, $0,0.3582,-0.0000,0.7597$

$\mathrm{O}, 0,0.2340,-0.0001,1.971$

$\mathrm{O}, 0,1.5802,0.000,0.1554$

$\mathrm{H}, 0,2.2309,-0.0000,0.887$

C, $0,-0.7596,0.0000,-0.2506$

$\mathrm{H}, 0,-1.6796,-0.0000,0.3417$

C, $0,-0.7062,1.2638,-1.1103$

$\mathrm{H}, 0,-0.7408,2.1660,-0.4932$

$\mathrm{H}, 0,0.2107,1.2848,-1.703$

$\mathrm{H}, 0,-1.5613,1.2855,-1.7917$

C, $0,-0.7062,-1.2636,-1.1105$

$\mathrm{H}, 0,0.2107,-1.2845,-1.7034$

$\mathrm{H}, 0,-0.7408,-2.1659,-0.4935$

$\mathrm{H}, 0,-1.5612,-1.2852,-1.7920$
Butanoic acid

C, $, 0,0.1019,1.6136,0.0194$

C, $0,0.1227,1.6238,1.5479$

H, $0,1.1172,1.6175,-0.3606$

$\mathrm{H}, 0,-0.3734,2.5220,-0.3396$

C, $0,-0.6498,0.4227,-0.5726$

$\mathrm{H}, 0,0.6431,2.5013,1.9177$

$\mathrm{H}, 0,0.6286,0.7493,1.9397$

$\mathrm{H}, 0,-0.8858,1.6442,1.9526$

C, $0,0.0617,-0.8966,-0.402$

$\mathrm{H}, 0,-0.795,0.5519,-1.6424$

$\mathrm{H}, 0,-1.6434,0.3270,-0.1462$

$\mathrm{O}, 0,1.1846,-1.0482,-0.0471$

$\mathrm{O}, 0,-0.7209,-1.9231,-0.7327$

$\mathrm{H}, 0,-0.2153,-2.7239,-0.632$

Oxalic acid

$\mathrm{C}, 0,-0.6220,-0.0868,0.4474$

$\mathrm{O}, 0,-0.7661,0.4366,1.515$

C, $0,0.6011,0.1218,-0.4674$

$\mathrm{O}, 0,0.7455,-0.4021,-1.5352$

$\mathrm{O}, 0,1.4667,0.9675,0.1063$

$\mathrm{H}, 0,2.2119,1.0636,-0.5059$

$\mathrm{O}, 0,-1.4880,-0.931,-0.1266$

$\mathrm{H}, 0,-2.2331,-1.0280,0.4856$

Succinic anhydride

C, $0,1.228172445$,

$0.1550063058,0.7754038115$

H,0,1.3011996117,

$0.0856408222,1.8530569548$

$\mathrm{H}, 0,2.2327590755$,

$0.1013357876,0.3761850462$

C, $0,0.4344387649$,

$1.3645903843,0.2877713389$

$\mathrm{H}, 0,0.0538868979$,

$1.9886207466,1.0860286517$

$\mathrm{H}, 0,0.9840965023$,

$2.0030769279,-0.3917070415$

C, $0,0.439117081,1.0331016703,0.2$ 652063688

C, $0,-0.7381455763$,

$0.7598072107,-0.4564032518$

O,0,0.6722216997,2.1749200846,0.

3992456376

$\mathrm{O}, 0,-1.6171363158$

$1.3112803858,-1.0034926983$

$\mathrm{O}, 0,-$

$0.6492651808,0.5934212595$,

0.4151820913
Esters

Methyl propanoate

C, $0,0.0423,0 .,-0.09939$

$\mathrm{C}, 0,0.0809,0 ., 1.4095$

$\mathrm{O}, 0,0.99228,0,-, 0.81348$

$\mathrm{O}, 0,-1.20614,0 .,-0.5488$

$\mathrm{C}, 0,-1.38274,0 .,-1.9536$

C, $0,1.49739,0 ., 1.97053$

$\mathrm{H}, 0,-0.47890,-0.86640,1.74889$

$\mathrm{H}, 0,-0.47890,0.86640,1.74889$

H, $0,1.4698,0.3 .05497$

$\mathrm{H}, 0,2.04640,-0.87441,1.64288$

$\mathrm{H}, 0,2.04640,0.87441,1.64288$

$\mathrm{H}, 0,-2.44909,0 .,-2.11716$

Propyl methanoate

C, $0,1.36183,0.00009,1.54114$

$\mathrm{H}, 0,1.35700,0.00013,2.62624$

$\mathrm{O}, 0,2.34618,0.0000,0.88341$

$\mathrm{O}, 0,0.12868,0.00006,1.08311$

C, $0,-0.04643,-0.00001,-0.3326$

C, $0,-1.53745,-0.00004,-0.61202$

C, $0,-1.83303,-0.0001,-2.11196$

$\mathrm{H}, 0,0.43771,-0.87518,-0.74581$

$\mathrm{H}, 0,0.43770,0.87510,-0.74591$

$\mathrm{H}, 0,-1.98287,-0.87160,-0.14282$

$\mathrm{H}, 0,-1.98289,0.87154,-0.14288$

$\mathrm{H}, 0,-2.90257,-0.00023,-2.29223$

$\mathrm{H}, 0,-1.41630,0.87725,-2.5979$

$\mathrm{H}, 0,-1.4162,-0.8774,-2.59785$

Isopropyl methanoate

$\mathrm{O}, 0,-0.86002,0.14804,0.11338$

$\mathrm{C}, 0,-0.85165,0.15738,1.42822$

C, $0,0.39385,0.14715,-0.59033$

$\mathrm{O}, 0,0.10580,0.15933,2.12610$

$\mathrm{H}, 0,-1.86987,0.16721,1.8037$

C, $0,0.93185,-1.27260,-0.67369$

$\mathrm{H}, 0,1.08253,0.76885,-0.03685$

C, $0,0.10829,0.7587,-1.94999$

$\mathrm{H}, 0,0.22494,-1.91618,-1.18717$

$\mathrm{H}, 0,1.11677,-1.67334,0.31492$

$\mathrm{H}, 0,1.8671,-1.2835,-1.22397$

$\mathrm{H}, 0,1.0213,0.81249,-2.53352$

$\mathrm{H}, 0,-0.28988,1.76115,-1.84294$

$\mathrm{H}, 0,-0.61336,0.16023,-2.4953$ 
$\alpha$-Lactone

$\mathrm{C}, 0,0.10325,-0.0002,0.315$ $\mathrm{O}, 0,0.6055,-0.0011,1.3715$

$\mathrm{O}, 0,0.4421,0.0036,-0.9423$

C, $0,-0.9990,-0.0020,-0.602$

$\mathrm{H}, 0,-1.5000,-0.9171,-0.8560$

$\mathrm{H}, 0,-1.5065,0.9113,-0.8514$

$\delta$-Lactone

C, $0,0.2595,0.0542,-1.3036$ $\mathrm{C}, 0,0.2860,0.0767,1.575$

C, $0,1.565,-0.1020,0.7892$

C, $0,1.4346,0.6403,-0.5309$

$\mathrm{H}, 0,0.529,-0.939,-1.6839$

$\mathrm{H}, 0,-0.0176,0.6548,-2.173$

$\mathrm{H}, 0,0.1546,1.1295,1.8532$

$\mathrm{H}, 0,0.273,-0.5215911,2.4885$

$\mathrm{H}, 0,2.4036,0.2760,1.3858$

$\mathrm{H}, 0,1.7445,-1.1683,0.6087$

$\mathrm{H}, 0,1.2633,1.7057,-0.3342$

, $0,1.2633,1.7057,0.3342$

C, $0,-1.0053,-0.1536,-0.4926$

$\mathrm{C}, 0,-1.0053,-0.1536,-0.4926$

$\mathrm{O}, 0,-2.0991,-0.2597,-1.0093$

$0,0,-0.8941,-0.3404,0.8541$

\section{Carboxyl Derivatives \\ Acetic Anhydride}

C, $0,2.2018,-0.7679,0.8916$

$\mathrm{H}, 0,2.4908,0.0882,1.4876$

$\mathrm{H}, 0,3.0478,-1.145,0.340$

$\mathrm{H}, 0,1.8194,-1.5240,1.5650$

C, $0,1.1216,-0.3593,-0.0593$

$\mathrm{O}, 0,1.1485,-0.3966,-1.2574$

$\mathrm{O}, 0,0.0462,0.1119,0.6496$

C, $0,-1.1822,0.5390,0.1324$

O, $0,-1.6878,1.5025,0.6166$

$\mathrm{C}, 0,-1.7615,-0.3320,-0.9439$

$\mathrm{H}, 0,-2.7423,0.0380,-1.1956$

$\mathrm{H}, 0,-1.1161,-0.3257,-1.8090$

$\mathrm{H}, 0,-1.832,-1.3518,-0.5843$

\section{Succinic Anhydride}

C, $0,1.18881,-0.15693,0.8269$

H, $0,1.05646,-0.08304,1.91088$

$\mathrm{H}, 0,2.2599,-0.11137,0.62311$

C, $0,0.46965,-1.35889,0.23358$

$\mathrm{H}, 0,0.1998,-2.13685,0.94982$

$\mathrm{H}, 0,1.04157,-1.83453,-0.5692$

C, $0,0.50245,1.0366,0.1952$

C, $0,-0.78776,-0.77586,-0.37769$

$\mathrm{O}, 0,0.83199,2.1938,0.20540$

$\mathrm{O}, 0,-1.76044,-1.34530,-0.79910$

$\mathrm{O}, 0,-0.67115,0.61342,-0.42917$
Amines

Methanimine

C, $0,0.0112,-0.5874,-0.0008$

$\mathrm{N}, 0,0.1078,0.6793,-0.0082$

$\mathrm{H}, 0,0.9253,-1.1835,-0.0707$

$\mathrm{H}, 0,-0.9257,-1.1568,0.0708$

$\mathrm{H}, 0,-0.8220,1.1097,0.0628$

Diethyl amine

$\mathrm{H}, 0,-0.1200,-0.2312,-1.1433$

$\mathrm{N}, 0,0.066,0.1281,-0.225$

C, $0,0.9736,-0.7667,0.4659$

$\mathrm{C}, 0,-1.1872,0.355,0.4659$

C, $0,2.2605,-0.9563,-0.3253$

C, $0,-2.08,1.2988,-0.3253$

$\mathrm{H}, 0,2.9509,-1.6024,0.2078$

$\mathrm{H}, 0,-3,0083,1.4918,0.2078$

$\mathrm{H}, 0,1.2055,-0.3259,1.4309$

$\mathrm{H}, 0,0.5207,-1.7416,0.6708$

$\mathrm{H}, 0,-0.9601,0.798,1.4309$

$\mathrm{H}, 0,-1.7241,-0.5760,0.6708$

$\mathrm{H}, 0,2.0619,-1.4126,-1.2918$

$\mathrm{H}, 0,2.7432,-0.001,-0.4985$

$\mathrm{H}, 0,-2.3417,0.8739,-1.2918$

$\mathrm{H}, 0,-1.5790,2.2431,-0.4985$

Dimethyl amine

N, $0,0.41172,0.01167,-0.42748$

$\mathrm{H}, 0,1.31611,-0.44463,-0.3253$ C, $0,0.27935,1.02776,0.60723$

$\mathrm{H}, 0,1.11596,1.72718,0.54065$

$\mathrm{H}, 0,-0.64336,1.58926,0.43589$

$\mathrm{H}, 0,0.24071,0.62325,1.63290$

C, $0,-0.63559,-0.99534,-0.32922$

$\mathrm{H}, 0,-1.59968,-0.52530,-0.54290$

$\mathrm{H}, 0,-0.46643,-1.77176,-1.07894$

$\mathrm{H}, 0,-0.70788,-1.47425,0.66201$
1-Penatamine

$\mathrm{H}, 0,0.5043,3.1542,-1.41396$ C, $0,0.5040,3.13882,-0.32759$ $\mathrm{H}, 0,1.5392,3.13960,0.0020$

C, $0,-0.2439,1.91804,0.20679$ $\mathrm{H}, 0,0.0473,4.06199,0.01576$ C, $0,0.3667,0.5931,-0.25375$

$\mathrm{H}, 0,-1.28356,1.96443,-0.11077$

$\mathrm{H}, 0,-0.25601,1.94927,1.29402$ $\mathrm{H}, 0,1.40764,0.55057,0.06344$ $\mathrm{H}, 0,0.37932,0.56490,-1.34232$

C, $0,-0.37651,-0.63130,0.28160$ C, $0,0.23360,-1.95172,-0.17672$ $\mathrm{H}, 0,-1.41712,-0.58992,-0.03903$ $\mathrm{H}, 0,-0.3888,-0.61797,1.36764$ $\mathrm{H}, 0,1.25879,-2.0142,0.17549$ $\mathrm{H}, 0,0.27622,-1.97143,-1.26863$ $\mathrm{N}, 0,-0.49719$,-

$3.0772307321,0.38739$

$\mathrm{H}, 0,-0.06040,-3 ., 0.14025$

$\mathrm{H}, 0,-1.43023,-3.10863,0.0223$

1-Hexanamine

$\mathrm{N}, 0,-1.9337,0 .,-3.3070$

C, $0,-1.8502,0 .,-1.85$

C, $0,-0.4345,0 .,-1.2720$

C, $0,-0.4132,0 ., 0.2575$

$\mathrm{C}, 0,0.9993,0,0.8444$

C, $0,1.0199,0 ., 2.3740$

C, $0,2.4344,0 ., 2.9526$

$\mathrm{H}, 0,-2.3923,-0.8689,-1.4933$

$\mathrm{H}, 0,-2.3923,0.8689156881,-1.4933$

$\mathrm{H}, 0,0.1031,-,-1.6433$

$\mathrm{H}, 0,0.1031,0.8718,-1.6433$

$\mathrm{H}, 0,-0.9544,0.8706,0.6245$

$\mathrm{H}, 0,-0.9544,-0.8706,0.624$

$\mathrm{H}, 0,1.5411,-0.8707,0.4786$

$\mathrm{H}, 0,1.5411,0.8707,0.4786$

$\mathrm{H}, 0,0.4800,0.8703,2.7411$

$\mathrm{H}, 0,0.4800,-0.8703,2.7411$

H, $0,2.4159,0.4 .0381$

$\mathrm{H}, 0,2.9895,-0.8769,2.6314$

$\mathrm{H}, 0,2.9895,0.8769,2.6314$

$\mathrm{H}, 0,-1.4737,-0.8048,-3.6882$

$\mathrm{H}, 0,-1.4737,0.8048,-3.6882$ 
Ethyl methyl amine

$\mathrm{N}, 0,-0.706546,-0.009482,0.209836$ $\mathrm{H}, 0,-0.698138,0.111394,1.222538$ C, $0,0.67086,0.032562,-0.267068$ H, $0,1.274129,-0.817570,0.101668$ $\mathrm{H}, 0,0.642081,-0.058433,-1.359019$ C, $0,1.330829,1.344333,0.121982$ H, $0,1.386930,1.444506,1.210577$ $\mathrm{H}, 0,2,349539,1.40243,-0.270792$ H, $0,0.753222,2.184633,0.268196$ $\mathrm{H}, 0,0.753222,2.184633,0.268196$ $\mathrm{C}, 0,-1.35707,-1.275135,-0.104669$ $\mathrm{H}, 0,-2.358515,-1.287540,0.332024$ $\mathrm{H}, 0,-1.46228,-1.359843,-1.189824$ $\mathrm{H}, 0,-0.80883,-2.163762,0.250696$

Ethyl dimethyl amine

$\mathrm{N}, 0,0.237497,0.051605,-0.486616$ C $, 0,0.235232,-0.027499,0.964293$ $\mathrm{H}, 0,1,261929,0.086853,1,295077$ $\mathrm{H}, 0,-0.328959,0.800830,1.407612$ C, $0,-0.293392,-1.353738,1.501512$ $\mathrm{H}, 0,0.229198,-2.182778,1.036951$ $\mathrm{H}, 0,-0.13555,-1.406190,2.574495$ $\mathrm{H}, 0,-1.355770,-1.479542,1.325541$ C, $0,-1.090861,0.077184,-1.060049$ H, $0,-1.017510,0.114601,-2.140826$ $\mathrm{H}, 0,-1.636679,-0.820775,-0.80274$ $\mathrm{H}, 0,-1.679063,0.940241,-0.730991$ C, $0,1.026419,1.170056,-0.955185$ $\mathrm{H}, 0,2.041300,1.088464,-0.583270$ $\mathrm{H}, 0,1.067644,1.161646,-2.03849$ H, $0,0.626598,2.139398,-0.640461$

Methyl isopropyl amine

$\mathrm{N}, 0,0.425787,-0.742831,0.508235$ $\mathrm{H}, 0,0.308237,-0.824773,1.518133$ C, $0,1.856770,-0.705429,0.222941$ $\mathrm{H}, 0,2.34291,-1.533099,0.744876$ $\mathrm{H}, 0,2.03055,-0.845164,-0.845774$ $\mathrm{H}, 0,2,34665,0.234809,0.527818$ C $, 0,-0.293359,0.455471,0.071573$ C, $0,-0.293359,0.455471,0.071573$
$\mathrm{H}, 0,0.216884,1.3748,0.41993$ $\mathrm{H}, 0,0.216884,1.3748,0.41993$
$\mathrm{C}, 0,-1.69108,0.42210,0.671882$ $\mathrm{C}, 0,-1.69108,0.42210,0.671882$
$\mathrm{H}, 0,-1.651330,0.41039,1.76634$ $\mathrm{H}, 0,-1.651330,0.41039,1.76634$
$\mathrm{H}, 0,-2.26817,1.299351,0.366996$ H, $0,-2.214318,-0.477918,0.338045$ C $0,-0.361723,0.499849,-1.448883$ C, $0,0.628409,0.589900,-1.901953$ $\mathrm{H}, 0,0.628409,0.589900,-1.901953$ $\mathrm{H}, 0,-0.828068,-0.417629,-1.81840$
$\mathrm{H}, 0,-0.955868,1.357088,-1.778750$
Triethyl amine

$\mathrm{N}, 0,0.156737,-0.220976,-0.23908$ C, $0,0.15104,-0.298938,1.22363$

$\mathrm{H}, 0,1.185233,-0.169339,1.559381$ $\mathrm{H}, 0,-0.422485,0.528702,1.685590$ C,0,-1.169834,-0.29566,-0.859522 $\mathrm{H}, 0,-1.012848,-0.366127,-1.94358$ $\mathrm{H}, 0,-1.63233,-1.243356,-0.561392$ C $0,0.966699,0.893377,-0.741602$ H, $0,0.684210,1.058537,-1788294$ $\mathrm{H}, 0,0.684210,1.058537,-1.788294$ $\mathrm{H}, 0,0.751621,1.841294,-0.211352$ $\mathrm{C}, 0,-0.358640,-1.637472,1.76252$ $\mathrm{H}, 0,-0.211945,-1.68510,2.847572$ $\mathrm{H}, 0,-1.42694,-1.78567,1.571850$ $\mathrm{H}, 0,0.187771,-2.46709,1.301254$ C, $0,-2.150226,0.856444,-0.570186$ $\mathrm{H}, 0,-1.746830,1.822550,-0.894459$ H, $0,-3.091593,0.691925,-1.107864$ $\mathrm{H}, 0,-2.389767,0.934683,0.496235$ C, $0,2.470997,0.609708,-0.688598$ $\mathrm{H}, 0,3.030016,1.44329,-1.130155$ $\mathrm{H}, 0,2.833311,0.481036,0.337035$ $\mathrm{H}, 0,2.705173,-0.303234,-1.245779$

Dipropyl amine

H, $0,-0.366518,1.001393,0.739359$ $\mathrm{N}, 0,-0.045586,0.408576,-0.002773$ C, $0,-1.10569,-0.50420,-0.382410$ C, $0,1.184911,-0.243324,0.399760$ C, $0,-2.361897,0.25286,-0.800990$ $\mathrm{C}, 0,2.25332,0.778500,0.774963$ $\mathrm{H}, 0,-0.750510,-1.099728,-1.21918$ $\mathrm{H}, 0,-1.353196,-1.210931,0.416839$ $\mathrm{H}, 0,1.537210,-0.839174,-0.437997$ $\mathrm{H}, 0,1.03491,-0.938944,1.232304$ $\mathrm{H}, 0,-2.702879,0.868931,0.029521$ $\mathrm{H}, 0,-2.102874,0.934387,-1.605239$ $\mathrm{H}, 0,1.889715,1.39199,1.597750$ $\mathrm{H}, 0,2.40253,1.44751,-0.06678$

C, $0,-3.493769,-0.67522,-1.239654$ $\mathrm{H}, 0,-3.785814,-1.348893,-0.43895$ $\mathrm{H}, 0,-4.372596,-0.108994,-1.53087$ $\mathrm{H}, 0,-3.196266,-1.282349,-2.08997$ C,0,3.57788,0.130185,1.17509

$\mathrm{H}, 0,4.31698,0.880680,1.43634515$ $\mathrm{H}, 0,3.454680,-0.524259,2,033448$ $\mathrm{H}, 0,3.985170,-0.46443,0.362264$
Dibutyl amine

$\mathrm{H}, 0,-0.92198,-0.732704,0.027709$ $\mathrm{N}, 0,-0.047935,-0.241636,0.026595$ C, $0,-0.012653,0.68115,1.144243$ C, $0,0.151651,0.383245,-1.266315$ $\mathrm{C}, 0,-0.22448,-0.047817,2.46715$ C, $0,0.106529,-0.64799,-2.389199$ $\mathrm{H}, 0,0.962385,1.160808,1.150177$ $\mathrm{H}, 0,-0.751255,1.48348,1.044531$ H, $0,1.126524,0.863198,-1.257935$ $\mathrm{H}, 0,-0.580295,1.17351,-1.463660$ $\mathrm{H}, 0,-1.191935,-0.549061,2.449947$ $\mathrm{H}, 0,0.525511,-0.828794,2.555576$ $\mathrm{H}, 0,-0.86,-1.146040,-2.380500$ $\mathrm{H}, 0,0.848609,-1.414624,-2.184659$ C. $0,-0.16123,0.879958,3.681455$ , $0,0.910333,1.662508,3.580556$ , $0,-0.910333,1.662508,3.580556$ $\mathrm{H}, 0,0.803737,1.382050,3.701269$ C, $0,0.346828,-0.041244,-3.772459$ $\mathrm{H}, 0,-0.396003,0.729945,-3.965272$ $\mathrm{H}, 0,1.313952,0.456947,-3.784201$ C, $0,0.300118,-1.07943,-4.893052$ $\mathrm{H}, 0,0.475988,-0.621328,-5.861441$ $\mathrm{H}, 0,1.055020,-1.846764,-4.747520$ $\mathrm{H}, 0,-0.667450,-1.57201,-4.930946$ $\mathrm{C}, 0,-0.37444,0.14365,5.003635$ $\mathrm{H}, 0,0.380354,-0.623484,5.150645$ $\mathrm{H}, 0,-0.322045,0.825638,5.846684$ $\mathrm{H}, 0,-1.346403,-0.34096,5.030100$
Tripropyl amine

$\mathrm{N}, 0,-0.01519,0.057696,0.040521$ C, $0,-0.023722,0.056690,1.494457$ $\mathrm{H}, 0,1.006807,0.11660,1.824407$ $\mathrm{H}, 0,-0.520343,0.94062,1.904567$ C, $0,-1.322456,0.09089,-0.595993$ $\mathrm{H}, 0,-1.165067,-0.060318,-1.66004$ $\mathrm{H}, 0,-1.890837,-0.76864,-0.258490$ C $0,0.940813,1,001145,-0.520789$ C, $0,0.633885,1.233933,-1.535252$ $1,0,033856,1.233933,-1.535252$ $\mathrm{H}, 0,0.941286,1.950621,0.021460$ C, $0,-0.63580,-1.201478,2.109576$ $\mathrm{H}, 0,-1.69614,-1.259984,1.883172$ $\mathrm{H}, 0,-0.169949,-2.070068,1.653730$ $\mathrm{C}, 0,-2.170073,1.355759,-0.398146$ $\mathrm{H}, 0,-1.60972,2.229846,-0.72019$ $\mathrm{H}, 0,-2.386079,1.500362,0.656622$ C, $0,2.362829,0.441459,-0.57567$ $\mathrm{H}, 0,2.696944,0.164906,0.419933$ $\mathrm{H}, 0,2.345946,-0.47414,-1.159059$ C, $0,-0.453340,-1.242290,3.627148$ $\mathrm{H}, 0,-0.90313,-2.134740,4.050449$ $\mathrm{H}, 0,0.59865,-1.242813,3.898461$ $\mathrm{H}, 0,-0.916562,-0.382820,4.10412$ C. $0,-3.485720,1.28207,-1.173953$ $\mathrm{H}, 0,-4.081447,2.177014,-1.02492$ $\mathrm{H}, 0,-3.308411,1.177688,-2.240854$ $\mathrm{H}, 0,-4.083095,0.432041,-0.855520$ C, $0,3.35502,1.434120,-1.181603$ $\mathrm{H}, 0,4.354,1.012678,-1.222935524$ H, $0,3.071564,1.707715,-2.194464$ $4,0,071564,1.707715,-2.194464$

Methyl propyl amine

$\mathrm{N}, 0,0.905235,-0.4012,0.31579$ $\mathrm{H}, 0,0.89445,-0.36213,1.31684$ C, $0,2.27335,-0.44724,-0.15342$ $\mathrm{H}, 0,2.79386,-1.27013,0.32308$ $\mathrm{H}, 0,2.28341,-0.62572,-1.22342$ $\mathrm{H}, 0,2.8369,0.46993,0.03620$

C, $0,0.14094,0.70937,-0.21834$ $\mathrm{H}, 0,0.15433,0.63106,-1.30244$ $\mathrm{H}, 0,0.59280,1.67543,0.02815$ C, $0,-1.30528,0.68925,0.27168$ $\mathrm{H}, 0,-1.31345,0.74193,1.35951$ $\mathrm{H}, 0,-1.79768,159373,-0.07737$ C $, 0,-2.09254,-0.53629,-0.18962$ $\mathrm{H}, 0,-1.62900,-1.45136,0.15733$ $\mathrm{H}, 0,-3.11168,-0.50544,0.18425$ $\mathrm{H}, 0,-2.13949,-0.5793,-1.274373$ 


\section{Cyclohexanamine}

C, $0,0.82146,0,1.71523$

$\mathrm{H}, 0,1.67746,0 ., 2.38469$

$\mathrm{H}, 0,-0.06703,0 ., 2.34437$

C, $0,0.82678,-1.26385,0.8504$

C, $0,0.82678,1.26385,0.8504$

$\mathrm{H}, 0,0.7634,-2.14826,1.47826$

$\mathrm{H}, 0,1.77383,-1.33036,0.31700$

$\mathrm{H}, 0,0.7634,2.14826,1.47826$

$\mathrm{H}, 0,1.77383,1.33036,0.31700$

C, $0,-0.32707,-1.25581,-0.15616$

$\mathrm{C}, 0,-0.32707,1.25581,-0.15616$

$\mathrm{H}, 0,-0.27861,-2.14236,-0.78560$

$\mathrm{H}, 0,-1.27930,-1.294,0.36723$

$\mathrm{H}, 0,-0.27861,2.14236,-0.78560$

$\mathrm{H}, 0,-1.27930,1.294,0.3672307841$

C, $0,-0.3105113849,0 .,-1.02902$

$\mathrm{N}, 0,-1.47478,0 .,-1.9044$

$\mathrm{H}, 0,0.62640,0 .,-1.59593$

H, $0,-1.46721,-0.8047,-2.50238$

H, $0,-1.46721,0.8047,-2.50238$
Azacyclohexane

C, $0,0.67921,0 ., 1.30582$

$\mathrm{H}, 0,0.64089,0 ., 2.39144$

$\mathrm{H}, 0,1.73282,0 ., 1.03020$

$\mathrm{C}, 0,0.01143,-1.26002,0.7472$

C, $0,0.01143,1.26002,0.7472$

C, $0,-0.04379,-1.21059,-0.77752$

C, $0,-0.04379,1.21059,-0.77752$

$\mathrm{N}, 0,-0.72527,0 .,-1.20291$

$\mathrm{H}, 0,-1.00320,-1.33483,1.12860$

$\mathrm{H}, 0,-1.00320,1.33483,1.12860$

$\mathrm{H}, 0,0.54718,-2.14918,1.06893$

$\mathrm{H}, 0,0.54718,2.14918,1.06893$

$\mathrm{H}, 0,0.97508,-1.2721,-1.17545$

$\mathrm{H}, 0,0.97508,1.2721,-1.17545$

$\mathrm{H}, 0,-0.59011,-2.06600,-1.16040$

$\mathrm{H}, 0,-0.59011,2.06600,-1.16040$ $\mathrm{H}, 0,-0.84172,0 .,-2.19677$

Methylhydrazine

$\mathrm{N}, 0,0.90378,-0.79329,0.11508$

$\mathrm{H}, 0,0.95292,-0.73207,1.11693$

H,0,1.85954,-0.6991,-0.17916

$\mathrm{N}, 0,0.2556,0.42845,-0.30561$

$\mathrm{H}, 0,0.23934,0.36648,-1.3058$

C, $0,-1.12197,0.39854,0.14625$

$\mathrm{H}, 0,-1.15010,0.50410,1.22617$

$\mathrm{H}, 0,-1.64703,1.24482,-0.28123$

$\mathrm{H}, 0,-1.63883,-0.52158,-0.12060$

1,1-Dimethylhydrazine

$\mathrm{N}, 0,0.839,-0.86739,0.59664$

$\mathrm{H}, 0,0.88178,-0.77513,1.59637$

$\mathrm{H}, 0,1.79578,-0.78147,0.30143$

$\mathrm{N}, 0,0.18165,0.32816,0.12670$

$\mathrm{C}, 0,-1.17641,0.29935,0.62630$

$\mathrm{H}, 0,-1.16695,0.34483,1.71029$

$\mathrm{H}, 0,-1.70638,1.17144,0.2628$

$\mathrm{H}, 0,-1.7125,-0.60062,0.32362$

C, $0,0.19792,0.28992,-1.32003$

$\mathrm{H}, 0,1.22280,0.328,-1.67404$

$\mathrm{H}, 0,-0.27094,-0.61044,-1.71815$

$\mathrm{H}, 0,-0.31775,1.16201,-1.70347$
1,2-Dimethylhydrazine

$\mathrm{N}, 0,-0.6889,-0.01286,0.20991$

$\mathrm{H}, 0,-0.64653,0.0320,1.21128$

$\mathrm{N}, 0,0.68891,0.01284,-0.20996$

$\mathrm{H}, 0,0.64651,-0.03217,-1.21132$

C, $0,-1.34416,1.19813,-0.2473$

$\mathrm{H}, 0,-1.44784,1.1672,-1.3272$

$\mathrm{H}, 0,-2.34058,1.2355,0.17738$

H, $0,-0.8024,2.10403,0.01796$

C $0,13441,1.19811,0.24734$

$\mathrm{H}, 0,2.34057,-1.2355,-0.17739$

$\mathrm{H}, 0,1.44791,-1.1670,1.32723$

$\mathrm{H}, 0,0.80245,-2.1040,-0.01783$

Methyldiazene

$\mathrm{N}, 0,0.34682,0.21420,0.37470$

$\mathrm{N}, 0,0.34598,-1.05004,0.3738$

, $0,1.02464,-1.32561,1.10706$

C, $0,-0.57994,0.74520,-0.62658$

$\mathrm{H}, 0,-0.00524,1.37937,-1.30622$

$\mathrm{H}, 0,-1.08753,-0.0535,-1.17499$

$\mathrm{H}, 0,-1.30189,1.37939,-0.10608$

Nitriles

Propanonitrile

C, $0,0.3608,0 ., 0.8128$

$\mathrm{N}, 0,0.2572,0 ., 1.943$

$\mathrm{C}, 0,0.4820,0 .,-0.6556$

$\mathrm{H}, 0,1.0573,-0.8727,-0.9417$

$\mathrm{H}, 0,1.0573,0.8727,-0.9417$

C, $0,-0.8848,0 .,-1.3481$

$\mathrm{H}, 0,-0.7488,0 .,-2.4234$

$\mathrm{H}, 0,-1.4575,-0.8782,-1.0758$

H, $0,-1.4575,0.8782,-1.0758$

\section{Organofluorine}

Fluoroethane

F, $0,-0.4527,0 .,-1.129$

C, $0,-0.5022,0 ., 0.2425$

C, $0,0.9040,0 ., 0.7984$

$\mathrm{H}, 0,0.8812,0 ., 1.8836$

$\mathrm{H}, 0,-1.0525,0.8797,0.5516$

$\mathrm{H}, 0,-1.0525,-0.8797,0.5516$

$\mathrm{H}, 0,1.4435,-0.8793,0.4650$

$\mathrm{H}, 0,1.4435,0.8793,0.4650$

1,1,2-Trifluoroethane

C, $0,0.4923,-0.7579,0.7074$ $\mathrm{H}, 0,0.5401,-0.7530,1.7810$ $\mathrm{H}, 0,1.466,-0.8400,0.263$

C, $0,-0.2670,0.434,0.1983$

H $, 0,-1,2963,0.4531,0.4892$

F, $0,0.5255,1.9591,0.9185$

$\mathrm{F}, 0,-0.4379,-2.3236,0.256$

1-Fluoroethene

C, $0,1.168,0 ., 0.232550$

H,0,1.18312,0.,1.302899

$\mathrm{H}, 0,2.101056,0 .,-0.290907$

C $0,0.045698,0,-0.430958$

$\mathrm{H}, 0,-0.039160,0 .,-1.495257$

F, $0,-1.169930,0 ., 0.185968$

1-Fluoropropane

F, $0,-0.6578,0,-,-1.7281$

C, $, 0,-0.7163,0 .,-0.3572$

C, $, 0,0.6871,0 ., 0.2128$

C, $0,0.6887,0,1.74$

H, $0,1.7028,0,2.1246$

$\mathrm{H}, 0,-1.2689,0.8799,-0.0500$

$\mathrm{H}, 0,-1.2689,-0.8799,-0.0500$

$\mathrm{H}, 0,1.2125,-0.872,-0.1641$

$\mathrm{H}, 0,1.2125,0.872,-0.1641$

$\mathrm{H}, 0,0.1866,0.8777,2.1382$

$\mathrm{H}, 0,0.1866,-0.8777,2.1382$

2-Fluoropropane

C, $0,-0.2917,0 ., 0.2372$

F, $0,0.8579,0 ., 1.0503$

$\mathrm{H}, 0,-1.1519,0 ., 0.9165$

C, $0,-0.2708,-1.2671,-0.5864$

$\mathrm{C}, 0,-0.2708,1.2671,-0.5864$

H, $0,-0,2295,-2,1418,0.0659$

$\mathrm{H}, 0,-0.2295,2.1418,0.0659$

$\mathrm{H}, 0,-1.1654,-1.3369,-1.2109$

$\mathrm{H}, 0,0.61,-1.2727,-1.2330$

$\mathrm{H}, 0,-1.1654,1.3369,-1.2109$

$\mathrm{H}, 0,0.61,1.2727,-1.2330$ 


\section{1-Fluorobutane}

C, $0,1.1014,0 .,-2.2516$

C, $0,1.0949,0 .,-0.7237$

$\mathrm{H}, 0,2.1154,0 .,-2.6383$

$\mathrm{H}, 0,0.5959,0.8771,-2.6448$

$\mathrm{H}, 0,0.5959,-0.8771,-2.6448$

C, $0,-0.319,0 .,-0.1399$

$\mathrm{H}, 0,1.6379,0.8708,-0.3623$

$\mathrm{H}, 0,1.6379,-0.8708,-0.3623$

$\mathrm{C}, 0,-0.3211,0 ., 1.3750$

$\mathrm{H}, 0,-0.8668,-0.8725,-0.4858$

$\mathrm{H}, 0,-0.8668,0.8725,-0.4858$

$\mathrm{F}, 0,-1.6141,0 ., 1.8357$

$\mathrm{H}, 0,0.1704,0.8799,1.7726$

$\mathrm{H}, 0,0.1704,-0.8799,1.7726$

\section{2-Fluorobutane}

$\mathrm{H}, 0,0.2941,2.0661,-1.3634$ C, $0,0.3004,2.0067,-0.2785$

$\mathrm{H}, 0,1.3284,1.895,0.044$

$\mathrm{C}, 0,-0.5532,0.8399,0.2151$

$\mathrm{H}, 0,-0.0751,2.9492,0.1062$

C, $0,-0.0665,-0.5079,-0.2887$

$\mathrm{H}, 0,-1.5841,0.9719,-0.1039$

$\mathrm{H}, 0,-0.5585,0.8192,1.3016$

F, $0,1.235,-0.68,0.1409$

$\mathrm{H}, 0,-0.0315,-0.5074,-1.3728$

C, $0,-0.8849,-1.6834,0.2066$

$\mathrm{H}, 0,-1.9069,-1.6173,-0.1518$

$\mathrm{H}, 0,-0.8997,-1.7046$,

H, $0,-0.4564,-2.6144$,-

0.1466935143

1-Fluoro-2-methylpropane

C, $0,-0.545,-0.005,-1.0422$

C, $0,-0.5319,-0.0180,0.477$

F, $0,0.7399,0.032,-1.5278$

$\mathrm{H}, 0,-1.063,0.8654,-1.4253$

$\mathrm{H}, 0,-1.0169,-0.896,-1.4397$

C, $0,0.1023,1.2602,1.0318$

C, $0,0.1691,-1.2699,1.0110$

$\mathrm{H}, 0,-1.5759,-0.0480,0.7869$

$\mathrm{H}, 0,1.1373,1.3422,0.718$

$\mathrm{H}, 0,0.0776,1.2643,2.116$

$\mathrm{H}, 0,-0.4235,2.1450,0.6846$

$\mathrm{H}, 0,1.2071,-1.2918,0.6978$

$\mathrm{H}, 0,-0.3090,-2.1754,0.6484$

H, $0,0.1442,-1.2936,2.0958$
2-Fluoro-2-methylpropane

$\mathrm{F}, 0,0,0 .,-1.3939$

C, $0,0 ., 0 .,-0.00$

$\mathrm{C}, 0,1.4574,0 ., 0.4305$

C, $, 0,-0.7287,1.2621,0.4305$

C, $0,-0.7287,-1.2621,0.4305$

$\mathrm{H}, 0,1.5382,0 ., 1.5124$

$\mathrm{H}, 0,1.9643,-0.878,0.0473$

$\mathrm{H}, 0,1.9643,0.878,0.0473$

$\mathrm{H}, 0,-0.7691,1.3321,1.5124$

$\mathrm{H}, 0,-0.2212,2.1404,0.0473$

$\mathrm{H}, 0,-1.7430,1.2618,0.0473$

$\mathrm{H}, 0,-0.7691,-1.3321,1.51242$

$\mathrm{H}, 0,-1.7430,-1.2618,0.0473$

$\mathrm{H}, 0,-0.2212,-2.1404,0.0473$

\section{Organochlorine}

3-Chloropropene

$\mathrm{C}, 0,0.8255,-0.5965,0.6669$

C, $0,0.8335,-0.58,2.0033$

H, $0,1.7589,-0.6107,0.1089$

$\mathrm{H}, 0,1.7609,-0.6076,2.5648$

$\mathrm{H}, 0,-0.0875,-0.5624,2.5781$

C, $0,-0.428,-0.5995,-0.137$

$\mathrm{H}, 0,-0.4754,-1.4599,-0.8077$

$\mathrm{H}, 0,-1.3092,-0.6010,0.5057$

$\mathrm{Cl}, 0,-0.5313,0.8558,-1.1850$

1-Chloro-2-methylpropane

$\begin{array}{llllll}\mathrm{H} & 0 & 0.85501 & 1.61076 & -2.4434\end{array}$

$\begin{array}{llllll}\text { C } & 0 & 0.85457 & 1.61145 & -1.35848\end{array}$

$\begin{array}{llllll}\mathrm{H} & 0 & 1.89021 & 1.61239 & -1.02885\end{array}$

$\begin{array}{llllll}\text { C } & 0 & 0.10261 & 0.38802 & -0.8202\end{array}$

$\begin{array}{lllllll}\mathrm{H} & 0 & 0.39728 & 2.53919 & -1.02832\end{array}$

$\begin{array}{lllll}\text { C } & 0 & 0.71879 & -0.90936 & -1.34642\end{array}$

$\begin{array}{lllll}\mathrm{H} & 0 & -0.92861 & 0.44526 & -1.15661\end{array}$

$\begin{array}{lllll}\text { C } & 0 & 0.09664 & 0.45468 & 0.70508\end{array}$

$\begin{array}{lllll}\mathrm{H} & 0 & 1.75416 & -1.00302 & -1.02841\end{array}$

$\begin{array}{llllll}\mathrm{H} & 0 & 0.70379 & -0.92195 & -2.43154\end{array}$

$\begin{array}{lllll}H & 0 & 0.17914 & -1.78002 & -0.99504\end{array}$

$\begin{array}{llllll}\text { H } & 0 & 1.08597 & 0.30164 & 1.11122\end{array}$

$\begin{array}{lllll}\text { Cl } & 0 & -0.95835 & -0.79278 & 1.46287\end{array}$

$\begin{array}{lllll}\mathrm{H} & 0 & -0.28065 & 1.40422 & 1.05223\end{array}$

1-Chloropentane

C, $0,-1.688722,-0.416844,-2.887718$

$\mathrm{C}, 0,-1.786479,-0.392556,-1.35998$

C, $0,-0.418223,-0.416857,-0.66957$

C, $0,-0.516857,-0.393384,0.858582$

C, $0,0.817263,-0.448628,1.586422$

$\mathrm{Cl}, 0,1.823182,1.050880,1.319745$

$\mathrm{H}, 0,-2.678860,-0.39745,-3.350986$

$\mathrm{H}, 0,-1.129546,0.446403,-3.261446$

$\mathrm{H}, 0,-1.176129,-1.31860,-3.237160$

$\mathrm{H}, 0,-2.382075,-1.24824,-1.018384$

$\mathrm{H}, 0,-2.332863,0.504082,-1.043338$

$\mathrm{H}, 0,0.174841,0.43951,-1.007142$

$\mathrm{H}, 0,0.130622,-1.314878,-0.982494$

$\mathrm{H}, 0,-1.094401,-1.263274,1.200684$

$\mathrm{H}, 0,-1.068243,0.493765,1.187708$

$\mathrm{H}, 0,1.43491,-1.279536,1.245675$

H,0,0.685761,-0.517099,2.664891
2-Chloropentane

C $, 0,-0.00960,0.70733,-2.22830$ C, $0,0.00817,0.68697,-0.71019$

H, $0,1.01516,0.74806,-2.60449$

$\mathrm{H}, 0,-0.54778,1.574,-2.60575$

$\mathrm{H}, 0,-0.48429,-0.19083,-2.62360$

$\mathrm{H}, 0,0.44437,1.60714,-0.32332$

C, $0,0.72981,-0.52196,-0$.

$\mathrm{Cl}, 0,-1.72118,0.74147,-0.10318$

C, $0,0.91325,-0.50650,1.38795$

$\mathrm{H}, 0,1.71503,-0.56019,-0.60875$

H, $0,0.20181,-1.42939,-0.4360$

C, $0,1.67718,-1.72743,1.89926$

$\mathrm{H}, 0,-0.06478,-0.45646,1.86916$

$\mathrm{H}, 0,1.44517,0.40570,1.6756$

$\mathrm{H}, 0,2.67391,-1.78932,1.45697$

H. $0,1.15047,-2.65230,1.65559$

$\mathrm{H}, 0,1.79819,-1.6927,2.98252$

3-Chloropentane

C, $0,-0.36714,0 .,-0.03809$

$\mathrm{Cl}, 0,1.18253,0 ., 0.94442$

$\mathrm{H}, 0,-1.15117,0 ., 0.7201$

C, $, 0,-0.44311,-1.27316,-0.87216$

C, $0,-0.44311,1.273168-0.87216$

C, $0,-0.52466,-2.5675,-0.06674$

C, $0,-0.52466,2.5675,-0.06674$

$\mathrm{H}, 0,-0.62487,-3.42672,-0.73049$ $\mathrm{H}, 0,-0.62487,3.42672,-0.73049$ $\mathrm{H}, 0,0.36705,-2.71400,0.5408$

$\mathrm{H}, 0,-1.38847,-2.5618,0.60116$

$\mathrm{H}, 0,0.36705,2.71400,0.5408$

Н $0,-1.38847,2.5618,0.60116$

$\mathrm{H}, 0,-1.33474,-1.18162,-1.50117$

$\mathrm{H}, 0,0.41317,-1.30130,-1.55033$

$\mathrm{H}, 0,-1.33474,1.18162,-1.50117$

$\mathrm{H}, 0,0.41317,1.30130,-1.55033$ 
1-Chloro-2-methylbutane $\mathrm{H}, 0,0.2562,2.950,-1.45133$ C, $0,0.2457,2.9457,-0.36739$ H, $0,1.27584,2.96406,-0.02302$ C, $0,-0.50222,1.73644,0.19628$ $\mathrm{H}, 0,-0.2267,3.86718,-0.04269$ C, $0,0.16796,0.38040,-0.09193$ $\mathrm{H}, 0,-1.5177,1.72623,-0.19557$ H, $0,-0.59141,1.85985,1.27297$ H, H, $, 1.17598,0.40801,0.31416$ $\mathrm{C}, 0,0.24974,0.07379,-1.58943$ C, $0,-0.60450,-0.69893,0.66588$ $\mathrm{Cl}, 0,0.22245,-2.29934,0.65981$ $\mathrm{H}, 0,-1.58183,-0.86297,0.23576$ $\mathrm{H}, 0,-0.72008,-0.43892,1.70656$ $\mathrm{H}, 0,0.86378,0.80211,-2.10574$ H, $0,-0.73914,0.09057,-2.04151$ $\mathrm{H}, 0,-0.73914,0.09057,-2.04151$
$\mathrm{H}, 0,0.68287,-0.90264,-1.76692$

1-Chloro-3-methylbutane

$\mathrm{H}, 0,0.5604,1.0562,-1.2700$ C, $0,0.531,1.005,-0.1982$

$\mathrm{H}, 0,1.5175,1.0668,0.2251$

C, $0,-0.2782,-0.1624,0.3439$

$\mathrm{Cl}, 0,-0.3020,2.6397,0.3143$

C, $0,0.3804,-1.5253,0.0251$

$\mathrm{H}, 0,-1.2764,-0.1374,-0.076$

H, $0,-0.3679,-0.0499,1.4174$ C, $0,0.3857,-1.818,-1.487$

C, $, 0,-0.3776,-2.6417,0.7697$

$\mathrm{H}, 0,1.4055,-1.5072,0.3838$

$\mathrm{H}, 0,-1.4103,-2.6791,0.4379$

H, $0,-0.3689,-2.4714,1.8407$

$\mathrm{H}, 0,0.9651,-1.088,-2.0417$

$\mathrm{H}, 0,-0.6293,-1.8124,-1.871$

$\mathrm{H}, 0,0.8150,-2.7949,-1.6813$

$\mathrm{H}, 0,0.0765,-3.6070,0.5753$
2-Chloro-2-methylbutane

$\mathrm{H}, 0,-0.1075,2.2752,-1.4014$

C, $0,-0.0503,2.240,-0.3206$

$\mathrm{H}, 0,0.9872,2.1563,-0.0276$

$\mathrm{C}, 0,-0.8681,1.0703,0.2511$

$\mathrm{H}, 0,-0.4395,3.1770,0.0623$

C, $0,-0.3660,-0.3297,-0.1113$

$\mathrm{H}, 0,-1.8913,1.134,-0.1115$

$\mathrm{H}, 0,-0.9018,1.1472,1.3307$

Cl, $0,1.373,-0.5338,0.6825$

C, $0,0.181,-0.5594,-1.6057$

$\mathrm{C}, 0,-0.181,-0.5594,-1.6057$

C, $0,-1.2332,-1.4111,0.5259$

$\mathrm{H}, 0,-2.2311,-1.3577,0.1009$

$\mathrm{H}, 0,-1.3009,-1.2677,1.5959$

$\mathrm{H}, 0,0.5327,0.1322,-2.0269$

$\mathrm{H}, 0,-1.1379,-0.4242,-2.102$ $\mathrm{H}, 0,0.166,-1.5667,-1.7910$ $\mathrm{H}, 0,-0.8239,-2.3931,0.3318$

2-Chloro-3-methylbutane

$\mathrm{H}, 0,0.4950,1.6890,-1.8193$

C, $0,0.5750,1.7032,-0.7385$

$\mathrm{H}, 0,1.6225,1.6319,-0.467$

C, $0,-0.2245,0.5570,-0.132$

$\mathrm{H}, 0,0.1965,2.6546,-0.3880$

C, $0,0.3095,-0.848,-0.4489$

$\mathrm{H}, 0,-1.2593,0.6297,-0.43$

$\mathrm{Cl}, 0,-0.2709,0.8137,1.6701$

C, $0,0.4668,-1.0424,-1.964$

C, $0,-0.5923,-1.9498,0.118$

$\mathrm{H}, 0,1.2913,-0.9342,0.009$

$\mathrm{H}, 0,-1.5863,-1.8983,-0.319$

$\mathrm{H}, 0,-0.6941,-1.8789,1.1930$

$\mathrm{H}, 0,1.2216,-0.3921,-2.3897$

$\mathrm{H}, 0,-0.4700,-0.8588,-2.4849$

$\mathrm{H}, 0,0.7624,-2.064,-2.1766$

H, $0,-0.1808,-2.9266,-0.1144$
1-Chloro-2,2-dimethylpropane $\mathrm{Cl}, 0,1.0358,0.8967,-1.5082$ C, $0,0.3592,0.9179,0.1628$ $\mathrm{H}, 0,1.2032,1.0867,0.8144$ $\mathrm{H}, 0,-0.2895,1.7799,0.2014$ C, $0,-0.3975,-0.354,0.5669$ $\mathrm{C}, 0,-0.8803,-0.1182,2.0091$ $\mathrm{H}, 0,-1.4293,-0.9821,2.3695$ $\mathrm{H}, 0,-0.0470,0.0485,2.6855$ $\mathrm{H}, 0,-1.5407,0.7418,2.0718$ $\mathrm{C}, 0,0.5275,-1.5777,0.5365$ $\mathrm{H}, 0,-0.0004,-2.4560,0.8949$ $\mathrm{H}, 0,0.8797,-1.7859,-0.4663$ $\mathrm{H}, 0,1.3953,-1.4298,1.1733$ C, $, 0,-1.6111,-0.5853,-0.3424$ $\mathrm{H}, 0,-2.276,0.2736,-0.3354$ H, $0,-1.3120,-0.7686,-1.3672$ $\mathrm{H}, 0,-2.1779,-1.4458,-0.0003$

Chlorocyclopropane

C, $0,-0.0572,-0.59276,-1.40367$ $\mathrm{C}, 0,-0.04876,-0.59451,0.08447$ C, $0,1.24414,-0.59276,-0.65243$ $\mathrm{H}, 0,-0.37214,-1.49417,-1.89916$ $\mathrm{H}, 0,-0.34643,0.31599,-1.89848$ $\mathrm{H}, 0,-0.35136,-1.47893,0.60867$ $\mathrm{H}, 0,1.81727,0.310 .6494483806$ $\mathrm{H}, 0,1.83072,-1.49417,-0.62751$ $\mathrm{Cl}, 0,-0.55335,0.85385,0.95857$

Chlorocyclobutane

C, $0,-0.16059,-0.190,-1.36543$ C, $0,-0.1497,-0.42189,0.14971$ C, $0,1.36543,-0.19020,0.16048$ C, $0,1.35307,-0.50924,-1.3532$ $\mathrm{H}, 0,-0.81865,-0.81411,-1.95676$ $\mathrm{H}, 0,-0.34593,0.85015,-1.60131$ $\mathrm{H}, 0,-0.4089,-1.43649,0.40887$ $\mathrm{H}, 0,1.9567,-0.81427,0.81846$ $\mathrm{H}, 0,1.60139,0.85005,0.34586$ $\mathrm{H}, 0,1.55120,-1.55612,-1.55140$ $\mathrm{H}, 0,1.98915,0.09259,-1.98929$ $\mathrm{Cl}, 0,-1.17495,0.62923,1.17507$
Chlorocyclopentane

C, $0,-1.30928,0.28401,-1.54291$ C, $0,-1.27800,-0.03008,-0.03447$ C, $0,0.14197,0.35207,0.36660$ C, $0,0.99767,-0.16956,-0.78182$ C, $0,0.16610,0.19389,-2.02736$ $\mathrm{H}, 0,-1.9566,-0.4029,-2.07493$ $\mathrm{H}, 0,-1.70172,1.28055,-1.71381$ $4,0,1.41989,-1.09118,0.14064$ $\mathrm{H}, 0,-2.0318,0.50239,0.53241$ H, $0,0.23740,1.42498,0.45690$ $\mathrm{H}, 0,1.99390,0.25550,-0.78956$ $\mathrm{H}, 0,1.09734,-1.2455,-0.686$

$\mathrm{H}, 0,0.48844,1.14690,-2.43256$

$\mathrm{H}, 0,0.29366,-0.54009,-2.81409$

$\mathrm{Cl}, 0,0.62874,-0.3007,1.97064$

Chlorocyclohexane

C, $0,2.26406,0 ., 0.12732$

$\mathrm{H}, 0,2.34669,0 ., 1.21263$

$\mathrm{H}, 0,3.27757,0 .,-0.26290$

C, $0,1.52650,-1.26324,-0.32458$

C, $0,1.52650,1.26324,-0.32458$

C, $0,0.0679,-1.2648,0.14949$

C, $0,0.0679,1.2648,0.14949$

C, $0,-0.64154,0,-0.32033$

$\mathrm{H}, 0,1.55410,-1.32919,-1.41085$ $\mathrm{H}, 0,1.55410,1.32919,-1.41085$ $\mathrm{H}, 0,2.02891,-2.14933,0.05158$ $\mathrm{H}, 0,2.02891,2.14933,0.05158$ H, $0,0.03211,-1.30626,1.2344$ $\mathrm{H}, 0,0.03211,1,30626,1.2344$ $\mathrm{H}, 0,-0.45350,-2.13992,-0.22185$ $\mathrm{H}, 0,-0.45350,2.13992,-0.22185$ $\mathrm{H}, 0,-0.72000,0 .,-1.39866$ $\mathrm{Cl}, 0,-2.35856,0 ., 0.25890$

1,1-Dichloroethane

C, $0,0.5269,0 ., 0.1563$

C $, 0,0.5222,0 ., 1.6687$

H, $0,1.5267,0,-0.2658$

$\mathrm{Cl}, 0,-0.2764,-1.4816,-0.494$

$\mathrm{Cl}, 0,-0.2764,1.4816,-0.494$

$\mathrm{H}, 0,-0.4990,0 ., 2.0503$

$\mathrm{H}, 0,1.0388,-0.8893,2.0358$

H, $0,1.0388,0.8893,2.0358$ 
1,2-Dichloroethane

$\mathrm{Cl}, 0,0.0964,1.1593,-1.3846$ C, $0,0.0313,1.09,0.4204$

$\mathrm{H}, 0,1.0591,1.143,0.7828$

C, $0,-0.6822,-0.1374,0.9435$

$\mathrm{H}, 0,-0.5101,1.9859,0.7478$

$\mathrm{Cl}, 0,0.2463,-1.6631,0.6663$

$\mathrm{H}, 0,-1.6581,-0.2630,0.4723$

$\mathrm{H}, 0,-0.8130,-0.0413,2.0249$

1,3-Dichloropropane
$\begin{array}{lllll}\text { C } & 0 & -0.6344 & -0.59701 & -0.10545\end{array}$
$\begin{array}{lllll}\text { C } & 0 & -0.73322 & -0.5302 & 1.41245\end{array}$
$\begin{array}{llllll}\text { C } & 0 & 0.75173 & -0.24645 & -0.62585\end{array}$
$\begin{array}{lllll}\mathrm{Cl} & 0 & -0.52925 & 1.14 & 2.04677\end{array}$
$\begin{array}{lllll}\text { Cl } & 0 & 0.82979 & -0.43928 & -2.40812\end{array}$
$\begin{array}{lllll}\mathrm{H} & 0 & -1.70509 & -0.86007 & 1.74375\end{array}$
$\begin{array}{lrrrr}\text { H } & 0 & 0.02229 & -1.1337 & 1.89285\end{array}$
$\begin{array}{cccccc}\mathrm{H} & 0 & 0.02229 & -1.1337 & 1.89285 \\ \mathrm{H} & 0 & 1.01434 & 0.77664 & -0.4142\end{array}$
$\begin{array}{lllll}\mathrm{H} & 0 & 1.01434 & 0.77664 & -0.4142 \\ \mathrm{H} & 0 & 1.50727 & -0.89832 & -0.21335\end{array}$
$\begin{array}{lllll}\mathrm{H} & 0 & -1.37252 & 0.05771 & -0.55222\end{array}$
$\begin{array}{lllll}\text { H } & 0 & -0.88019 & -1.61255 & -0.40082\end{array}$

Chloroethene

C, $0,-0.7407,0,0.1510$

C, $0,-0.7474,0 ., 1.4824$

$\mathrm{Cl}, 0,0.7115,0 .,-0.7896$

$\mathrm{H}, 0,-1.6404,0 .,-0.45$

$\mathrm{H}, 0,0.1663,0 ., 2.0644$

$\mathrm{H}, 0,-1.6935,0 ., 2.010$

Trichloroethene

C, $0,-0.7229,0 ., 0.8491$

$\mathrm{H}, 0,-0.7614,0 ., 1.9137$

C, $0,0.3912,0 ., 0.1846$

$\mathrm{Cl}, 0,1.9644,0 ., 1.05163$

$\mathrm{Cl}, 0,0.533,0 .,-1.5898$

$\mathrm{Cl}, 0,-2.3356,0 ., 0.0607$
Tetrachloroethene

C, $0,0.0013,0 .,-0.6495$

C, $0,-0.0013,0 ., 0.6495$

$\mathrm{Cl}, 0,1.4892,0 ., 1.6276$

$\mathrm{Cl}, 0,-1.4964,0 ., 1.6210$

$\mathrm{Cl}, 0,-1.4892,0 .,-1.6276$

$\mathrm{Cl}, 0,1.4964,0 .,-1.6210$

Chlorofluoromethane

C, $0,-0.770259,-0.225587,-0.066590$

$\mathrm{H}, 0,-1.370281,-0.212562,0.825850$

H, $0,-0.98626,-1.07728,-0.686755$

$\mathrm{Cl}, 0,0.930545,-0.321302,0.419928$

$\mathrm{F}, 0,-0.982352,0.900613,-0.764259$

Chlorodifluoromethane

C, $0,0.001547,-0.004931,-0.002190$

$\mathrm{H}, 0,-0.014272,0.013335,1.070660$

Cl, $0,1.670186,-0.015835,-0.558305$

$\mathrm{F}, 0,-0.619537,-1.082348-0.446314$

$\mathrm{F}, 0,-0.617451,1.058092,-0.482758$

Dichlorofluoromethane

$\mathrm{C}, 0,0.008613,-0.000354,-0.014940$

$\mathrm{H}, 0,-0.011783,0.024890,1.056863$

$\mathrm{Cl}, 0,1.685562,-0.046379,-0.542594$

F, $0,-0.610958,-1.093737,-0.446256$

$\mathrm{Cl}, 0,-0.837204,1.415422,-0.625285$
Organobromine

Bromomethane

$\mathrm{C}, 0,0 ., 0 .,-1.5267$

$\mathrm{Br}, 0,0,0 ., 0.4206$

$\mathrm{H}, 0,1.0255,0 .,-1.8537$

$\mathrm{H}, 0,-0.5127,-0.8881,-1.8537$

$\mathrm{H}, 0,-0.5127,0.8881,-1.8537$

Bromoethane

$\mathrm{Br}, 0,-0.1719,0 .,-0.7761$

C, $0,-0.355,0 ., 1.1800$

C, $0,1.0007,0.1 .8587$

$\mathrm{H}, 0,0.8628,0 ., 2.9367$

$\mathrm{H}, 0,-0.9315,0.880,1.4113$

$\mathrm{H}, 0,-0.9315,-0.880,1.4113$

$\mathrm{H}, 0,1.5722,-0.8786,1.5865$

$\mathrm{H}, 0,1.5722,0.8786,1.5865$

1-Bromopropane

$\mathrm{Br}, 0,-0.3590,0 .,-1.2509$

$\mathrm{C}, 0,-0.4184,0 ., 0.7515$

C, $0,0.9850,0 ., 1.3473$

C, $0,0.9022,0 ., 2.887$

$\mathrm{H}, 0,1.8957,0.3 .3205$

$\mathrm{H}, 0,-0.9723,0.8831,1.0203$

$\mathrm{H}, 0,-0.9723,-0.8831,1.0203$

$\mathrm{H}, 0,1.5230,-0.8747,1.0050$

$\mathrm{H}, 0,1.5230,0.8747,1.0050$

$\mathrm{H}, 0,0.3778,0.8795,3.2451$

$\mathrm{H}, 0,0.3778,-0.8795,3.2451$

2-Bromopropane

C, $0,-0.94017,0 .,-0.375$

$\mathrm{Br}, 0,0.72,0 ., 0.7097$

H, $0,-1.7199,0.0 .3696$

C, $0,-0.9871,-1.2716,-1.20$

C, $0,-0.9871,1.2716,-1.20$

$\mathrm{H}, 0,-0.9413,-2.152,-0.5775$

$\mathrm{H}, 0,-0.9413,2.152,-0.5775$

$\mathrm{H}, 0,-1.9174,-1.2985,-1.7666$

$\mathrm{H}, 0,-0.1626,-1.3068,-1.9069$

$\mathrm{H}, 0,-1.9174,1.2985,-1.7666$

H, $0,-0.1626,1.3068,-1.9069$
1-Bromobutane

C, $0,1.9843,0 .,-3.1113$

C, $0,1.9764,0 .,-1.5826$

$\mathrm{H}, 0,3.0026,0 .,-3.5012$

$\mathrm{H}, 0,1.4765,0.8808,-3.5088$

$\mathrm{H}, 0,1.4765,-0.8808,-3.5088$

C, $0,0.5572,0 .,-1.001$

$\mathrm{H}, 0,2.5202,0.8750,-1.2146$

$\mathrm{H}, 0,2.5202,-0.8750,-1.2146$

C, $0,0.5761,0.0 .5147$

$\mathrm{H}, 0,0.0118,-0.8762,-1.3587$

$\mathrm{H}, 0,0.0118,0.8762,-1.3587$

$\mathrm{Br}, 0,-1.2482,0 ., 1.2828$

$\mathrm{H}, 0,1.0522,0.8861,0.9244$

$\mathrm{H}, 0,1.0522,-0.8861,0.9244$

2-Bromobutane

$\mathrm{H}, 0,-0.5523,2.4216,-1.552$

C. $0,-0.5158,2.3983,-0.4613$

$\mathrm{H}, 0,0.5309,2.3717,-0.1618$

C, $0,-1.281,1.1990,0.0898$

$\mathrm{H}, 0,-0.9476,3.330,-0.0951$

C, $0,-0.8209,-0.1583,-0.4196$

H, $0,-2.3379,1.2861,-0.1913$

$\mathrm{H}, 0,-1.2550,1.1981,1.1820$

$\mathrm{Br}, 0,1.057,-0.5274,0.1862$

$\mathrm{H}, 0,-0.7156,-0.1461,-1.502$

C, $0,-1.7039,-1.3085,0.0247$

$\mathrm{H}, 0,-2.7158,-1.1548,-0.3602$

$\mathrm{H}, 0,-1.7589,-1.3650,1.1117$

$\mathrm{H}, 0,-1.3385,-2.2623,-0.3505$

2-Bromo-2-methylpropane

$\mathrm{Br}, 0,0,0,-1.1772$

C, $0,0 ., 0 ., 0.8548$

C,0,1.4570,0.,1.2945

C, $0,-0.7285,1.2618,1.2945$

C, $0,-0.7285,-1.2618,1.2945$

$\mathrm{H}, 0,1.4980,0 ., 2.3877$

$\mathrm{H}, 0,1.9815,-0.8836,0.9349$

$\mathrm{H}, 0,1.9815,0.8836,0.9349$

$\mathrm{H}, 0,-0.7490,1.2973,2.3877$

$\mathrm{H}, 0,-0.225,2.1578,0.9349$

$\mathrm{H}, 0,-1.7560,1.2742,0.9349$

$\mathrm{H}, 0,-0.7490,-1.2973,2.3877$

$\mathrm{H}, 0,-1.7560,-1.2742,0.9349$

$\mathrm{H}, 0,-0.225,-2.1578,0.9349$ 
1,2-Dibromoethane

$\mathrm{Br}, 0,0.01229,1.30240,-1.29480$ C, $0,-0.06985,1.24813,0.65189$ $\mathrm{H}, 0,0.94695,1.29012,1.0027$

C, $0,-0.79636,0.02475,1.16313$ $\mathrm{H}, 0,-0.59965,2.14034,0.94916$ $\mathrm{Br}, 0,0.20267,-1.6183,0.84435$ $\mathrm{H}, 0,-1.7549,-0.10284,0.6901$ $\mathrm{H}, 0,-0.91923,0.09161,2.2334$

1,2-Dibromopropane

$\begin{array}{llll}\text { C } 0 & 0.9338 & 0.04482 & 0.07685\end{array}$ Br $0 \quad 1.59879-0.42435$

1.52247

$\begin{array}{lllll}\text { H } & 0 & 1.4051 & 0.97519 & 0.34484\end{array}$

$\begin{array}{lllll}\mathrm{H} & 0 & 1.21953 & -0.72094 & 0.78037\end{array}$

$\begin{array}{lllll}\mathrm{C} 0 & -0.57985 & 0.19904 & 0.01371\end{array}$

$\begin{array}{lllll}\mathrm{C} & 0 & 0.5384 & 0.1994 & 0.01371\end{array}$

$\begin{array}{lllll}\mathrm{H} 0 & -0.83846 & 0.94354 & -0.72267\end{array}$

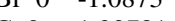

$\begin{array}{llll}\text { C } 0 & -1.33781 & -1.09462 & -0.2342\end{array}$

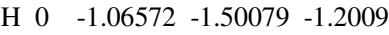

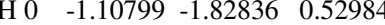

$\begin{array}{lllll}\text { H } & 0 & -2.40471 & -0.91268 & -0.22528\end{array}$

1-Bromoethene

C, $0,-1.0986,0 ., 0.511290$

C, $0,-1.0996,0.1 .833538$

$\mathrm{Br}, 0,0.49747,0 .,-0.5357$

$\mathrm{H}, 0,-1.98741,0 .,-0.10440$

$\mathrm{H}, 0,-2.04503,0 ., 2.3657870$

H,0,-0.1893256,0.,2.4196138

3-Bromo-1-propene

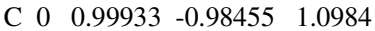

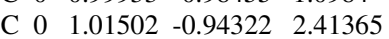

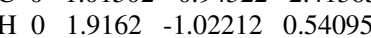

$\begin{array}{lllll}\text { H } & 0 & 1.93574 & -0.95432 & 2.96358\end{array}$

$\begin{array}{lllll}\text { H } & 0 & 0.11187 & -0.89477 & 2.993\end{array}$

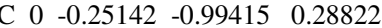

$\begin{array}{lllll}\text { H } & 0 & -0.27513 & -1.80086 & -0.42383\end{array}$

$\begin{array}{lllll}\text { H } & 0 & -1.14029 & -0.99809 & 0.89403\end{array}$

$\begin{array}{llll}\text { Br } 0 & -0.37503 & 0.6629 & -0.85055\end{array}$
Organosilicon

Dimethylsilane

$\mathrm{Si}, 0,0 ., 0 ., 0.55086$

$\mathrm{H}, 0,1.19405,0 ., 1.4273$

$\mathrm{H}, 0,-1.19405,0 ., 1.4273$

C, $0,0 ., 1.56150,-0.51303$

$\mathrm{C}, 0,0,-,-1.56150,-0.51303$

$\mathrm{H}, 0,0 ., 2.45719,0.10157$

$\mathrm{H}, 0,0,-,-2.45719,0.10157$

H,0,0.87720,1.6014,-1.15339

$\mathrm{H}, 0,-0.87720,1.6014,-1.15339$

$\mathrm{H}, 0,-0.87720,-1.6014,-1.15339$

$\mathrm{H}, 0,0.87720,-1.60143,-1.15339$

Dimethylsilyl chloride

Si, $0,-0.55834,0 ., 0.00294$

$\mathrm{Cl}, 0,1.18940,0 ., 1.11929$

$\mathrm{H}, 0,-1.67995,0 ., 0.97633$

C, $0,-0.59255,-1.54910,-1.0401$

C, $0,-0.59255,1.54910,-1.0401$

$\mathrm{H}, 0,-0.54174,-2.44728,-0.42009$

$\mathrm{H}, 0,-0.54174,2.44728,-0.42009$

$\mathrm{H}, 0,-1.51555,-1.59380,-1.62660$

$\mathrm{H}, 0,0.25105,-1.57000,-1.73514$

$\mathrm{H}, 0,-1.51555,1.59380,-1.62660$

$\mathrm{H}, 0,0.25105,1.57000,-1.73514$

Trimethylsilane

$\mathrm{H}, 0,0,, 0,-,-1.87596$

$\mathrm{Si}, 0,0 ., 0 .,-0.38184$

$\mathrm{C}, 0,1.78242,0 ., 0.2242$

$\mathrm{C}, 0,-0.8912,1.5436,0.2242$

C, $0,-0.89121,-1.5436,0.2242$

$\mathrm{H}, 0,1.82302,0.1 .31770$

$\mathrm{H}, 0,-0.91151,1.57878,1.31770$

$\mathrm{H}, 0,-0.91151,-1.57878,1.31770$

$\mathrm{H}, 0,2.32308,-0.8830942,0.12783$

$\mathrm{H}, 0,2.32308,0.88309,-0.12783$

$\mathrm{H}, 0,-0.39676,2.45340,-0.12783$

$\mathrm{H}, 0,-1.9263,1.57030,-0.12783$

$\mathrm{H}, 0,-1.9263,-1.57030,-0.12783$

$\mathrm{H}, 0,-0.39676,-2.45340,-0.12783$

Trimethylsilylchloride

$\mathrm{Cl}, 0,0 ., 0 .,-1.75353$

$\mathrm{Si}, 0,0 ., 0 ., 0.32958$

C, $0,1.7842,0 ., 0.89271$

, $, 0,-0.89210,1.54517,0.89271$

C, $0,-0.89210,-1.54517,0.89271$

$\mathrm{H}, 0,1.84062,0 ., 1.98584$

$\mathrm{H}, 0,2.31399,-0.88374,0.52824$

, $0,2.31399,0.88374,0.52824$

$4,0,-0.92031,1.5940,1.98584$

, $0,-0.39164,2.44585,0.52824$

, $, 0,-1.92234,1.56210,0.52824$

$\mathrm{H}, 0,-0.92031,-1.59402,1.9858$

H, $0,-1.92234,-1.56210,0.52824$

H,0,-0.39164,-2.44585, 0.52824 
1-Hexanethiol

S, $,-1.61094,0 .,-3.15897$

C, $0,-1.6464,0 .,-1.32263$

C, $0,-0.21911,0 .,-0.78263$

C, $0,-0.17298,0 ., 0.74691$

C, $0,1.25035,0 ., 1.3084$

C, $0,1.30419,0 ., 2.83675$

C, $0,2.72886,0.3 .38959$

$\mathrm{H}, 0,-2.18315,-0.88478,-0.98228$

$\mathrm{H}, 0,-2.18315,0.88478,-0.98228$

H, $0,0.31199,-0.87623,-1.16506$

$\mathrm{H}, 0,0.31199,0.8762,-1.16506$

$\mathrm{H}, 0,-0.71084,0.87542,1.12584$

$\mathrm{H}, 0,-0.71084,-0.87542,1.12584$

$\mathrm{H}, 0,1.78913,-0.87477,0.92867$

H, $0,1.78913,0.87477,0.92867$

H, $0,0.76584,0.87422,3.21595$

, $0,0.76584,0.87422,3.21595$

$4,0,0.76584,-0.87422,3.21595$

$\mathrm{H}, 0,2.73438,0 ., 4.4805$

$\mathrm{H}, 0,3.28229,-0.88057,3.05654$

$\mathrm{H}, 0,3.28229,0.88057,3.05654$

$\mathrm{H}, 0,-2.93897,0 .,-3.35504$

1-heptanethiol

S, $0,-1.031764,3.65775,-1.858409$

C, $0,-0.975724,1.840390,-1.709544$

C, $0,-0.229880,1.329265,-0.479171$

C, $0,-0.25528,-0.198383,-0.380725$

C, $0,0.490276,-0.730398,0.84489$

C, $0,0.465827,-2.256245,0.950173$

C, $0,1.210945,-2.790173,2.1749$

$\mathrm{H}, 0,-0.555262,1.426504,-2.617648$

$\mathrm{H}, 0,-2.015801,1.53787,-1.67376$

$\mathrm{H}, 0,0.801413,1.672845,-0.514370$

$\mathrm{H}, 0,-0.67121,1.765934,0.411611$

$\mathrm{H}, 0,-1.288411,-0.53992,-0.349541$

$\mathrm{H}, 0,0.180935,-0.626909,-1.28131$

$\mathrm{H}, 0,1.523275,-0.388392,0.813382$

$\mathrm{H}, 0,0.054427,0.30034,1744799$

$\mathrm{H}, 0,0.567251,-2.598613,0.981726$

$\mathrm{H}, 0,-0.567251,-2.598613,0.981726$

$\mathrm{H}, 0,0.901320,-2.686651,0.049809$

$\mathrm{H}, 0,2.243959,-2.450177,2.144164$

$\mathrm{H}, 0,0.776047,-2.362062,3.075552$

$\mathrm{H}, 0,0.272044,3.869217,-1.990897$

C, $0,1.182175,-4.314983,2.273786$

$\mathrm{H}, 0,1.718245,-4.662650,3.151443$

$\mathrm{H}, 0,0.162756,-4.684402,2.341149$

$\mathrm{H}, 0,1.641772,-4.77323,1.402558$ 1-octanethiol

S, $0,-1.359066,4.128751,-2.154480$ C, $0,-1.281323,2.311714,-2.011296$

C, $0,-0.479021,1.805261,-0.814980$

C, $0,-0.487295,0.277317,-0.718171$

C, $0,0.31472,-0.250015,0.473342$

C, $0,0.307551,-1.776305,0.576850$

C, $0,1.109276,-2.304720,1.767638$

$\mathrm{H}, 0,-0.896783,1.902674,-2.93733$

$\mathrm{H}, 0,-2.316309,2.000314,-1.931410$

$\mathrm{H}, 0,0.546872,2.157642,-0.893852$

$\mathrm{H}, 0,-0.885331,2.236953,0.094690$

$\mathrm{H}, 0,-1.515222,-0.07301,-0.643229$

$\mathrm{H}, 0,-0.08661,-0.146243,-1.637438$

$\mathrm{H}, 0,1.34247,0.100790,0.398038$

$\mathrm{H}, 0,-0.085607,0.175143,1.391860$

$\mathrm{H}, 0,-0.720519,-2.126585,0.651829$

$\mathrm{H}, 0,0.707270,-2.200939,-0.342525$

$\mathrm{H}, 0,2.137721,-1.954951,1.693195$

$\mathrm{H}, 0,0.70999,-1.880388,2.687429$

$\mathrm{H}, 0,-0.063953,4.351485,-2.342229$

C, $0,1.102868,-3.830883,1.872846$

$\mathrm{H}, 0,0.075936,-4.182017,1.949002$

$\mathrm{H}, 0,1.503005,-4.256589,0.955029$

C, $0,1.905764,-4.351159,3.064647$

$\mathrm{H}, 0,1.50839,-3.970452,4.001341$

$\mathrm{H}, 0,1.882191,-5.435502,3.11215$

H, $0,2.946294,-4.04559,2.999886$ 1-nonanethiol

S, $0,-1.496239,4.671053,-2.452821$

C, $0,-1.416252,2.853918,-2.312081$

C, $0,-0.633679,2.346981,-1.102969$

$\mathrm{C}, 0,-0.639638,0.818817,-1.0095$

C, $0,0.142770,0.29099,0.19476$

C, $0,0.137788,-1.235515,0.294920$

C, $0,0.920009,-1.764326,1.498622$

$\mathrm{H}, 0,-1.014513,2.447847,-3.232108$

$\mathrm{H}, 0,-2.451659,2.539689,-2.25096$

$\mathrm{H}, 0,0.392509,2.702181,-1.163140$

H, $0,-1.056968,2.77568,-0.199647$

$\mathrm{H}, 0,-1.667799,0.465676,-0.953323$

H, $0,-0.221821,0.398260,-1.922505$

$\mathrm{H}, 0,1.170753,0.644654,0.138204$

$\mathrm{H}, 0,-0.274700,0.713182,1.107009$

$\mathrm{H}, 0,-0.890502,-1.58858,0.351098$

$\mathrm{H}, 0,0.554640,-1.657114,-0.618195$

H, $0,1.948310,-1.411066,1.442372$

$\mathrm{H}, 0,0.5032,-1.342321,2.411566$

$\mathrm{H}, 0,-0.198621,4.897511,-2.6173$

C, $0,0.915261,-3.290680,1.599763$

H, $0,-0.112868,-3.644724,1.656390$

$\mathrm{H}, 0,1.332192,-3.713514,0.687061$

C $0,1.697173,-3.820743,2.803064$

$\mathrm{H}, 0,1.280857,-3.400514,3.716178$

$\mathrm{H}, 0,2.725318,-3.469275,2.747303$

C, $0,1.687773,-5.346123,2.897311$

$\mathrm{H}, 0,2.129911,-5.796359,2.012943$

$\mathrm{H}, 0,2.249850,-5.690972,3.759751$

$\mathrm{H}, 0,0.674493,-5.727047,2.9892$ 1-decanethiol

S, $0,-3.279384,0.049656,-5.155755$ C, $0,-3.117178,-0.00755,-3.339561$

C, $0,-1.676926,-0.00137,-2.832650$

$\mathrm{C}, 0,-1.602053,-0.00275,-1.303450$ C, $, 0,-0.1658,0.000077,-0.775705$

C, $0,-0.084854,0.001786,0.751974$

$\mathrm{C}, 0,1.350736,0.004369,1.280675$

H, $0,-3.656110,-0.86936,-2965962$

H, $0,-3.644306,0.872975,-2.991173$

$\mathrm{H}, 0,-1.154190,-0.87214,-3.221701$

$\mathrm{H}, 0,-1.163,0.869859,-3.228544$

$\mathrm{H}, 0,-2.129191,0.867527,-0.916514$

$\mathrm{H}, 0,-2.126200,-0.87450,-0.915730$

$\mathrm{H}, 0,0.36079,-0.870276,-1.163121$

$\mathrm{H}, 0,0.357835,0.871141,-1.16514$

$\mathrm{H}, 0,-0.612035,0.872233,1.138779$

$\mathrm{H}, 0,-0.609713,-0.869208,1.140818$

$\mathrm{H}, 0,1.877812,-0.866103,0.893663$

$\mathrm{H}, 0,1.875437,0.875210,0.891370$

$\mathrm{H}, 0,-2.69056,-1.108723,-5.427173$

C, $0,1.432548,0.006471,2.808345$

H, $0,0.905419,0.876994,3.195269$

$\mathrm{H}, 0,0.907650,-0.864378,3.19761$

C, $0,2.867847,0.00903,3.337435$

$\mathrm{H}, 0,3.393448,0.879853,2.948811$

$\mathrm{H}, 0,3.395709,-0.861498,2.951195$ C, $0,2.950893,0.011229,4.864973$

$\mathrm{H}, 0,2.427582,-0.859231,5.255092$

$\mathrm{H}, 0,2.425379,0.881425,5.252685$

$\mathrm{C}, 0,4.387756,0.013776,5.385710$

H $0,4.928169,0.890926,5.040950$

$\mathrm{H}, 0,4.413492,0.015308,6.47108$

$\mathrm{H}, 0,4.930417,-0.862942,5.043370$ 
Butyl ethyl sulfide

C, $0,3.69053,-1.68758,0.50915$

$\mathrm{H}, 0,3.7298,-1.5100,1.58782$

$\mathrm{H}, 0,4.70853,-1.60772,0.11803$

$\mathrm{H}, 0,3.3494,-2.71492,0.35167$

C, $0,2.75421,-0.69456,-0.17059$

$\mathrm{H}, 0,2.75166,-0.87204,-1.25287$

$\mathrm{H}, 0,3.12975,0.32543,-0.02431$

C $0,1.32571,-0.78569,0.35783$

H, $0,0.94505,-1.80304,0.20845$

H, $0,1.32376,-0.60356,1.4390$

C, $0,0.39486,0.20841,-0.32466$

$\mathrm{H}, 0,0.37671,0.02149,-1.4041$

$\mathrm{H}, 0,0.75817,1.22966,-0.16462$

S, $0,-1.28630,0.05980,0.33756$

C, $0,-2.0994,1.33814,-0.65821$

$\mathrm{H}, 0,-1.60715,2.29961,-0.48108$

$\mathrm{H}, 0,-1.98816,1.09286,-1.71914$

C, $0,-3.57422,1.42635,-0.29032$

H, $0,-4.06693,2.19672,-0.88958$

$\mathrm{H}, 0,-4.08043,0.47576,-0.47378$

$\mathrm{H}, 0,-3.69942,1.68253,0.76429$

Methyl pentyl sulfide

S, $0,-0.77939,0,-,-2.32526$

C, $0,-0.8384,0 .,-0.50136$

C, $0,0.57377,0 ., 0.0777$

C, $0,0.58382,0 ., 1.60927$

C, $0,1.99369,0 ., 2.20286$

$\mathrm{C}, 0,2.00342,0 ., 3.73142$

$\mathrm{H}, 0,-1.38687,-0.88376,-0.16859$

$\mathrm{H}, 0,-1.38687,0.88376,-0.16859$

H $0,1.11476,-0.87591,-0.29077$

$\mathrm{H}, 0,1.11476,0.87591,-0.29077$

$\mathrm{H}, 0,0.03808,0.87549,1.9767$

$\mathrm{H}, 0,0.03808,-0.87549,1.9767$

$\mathrm{H}, 0,2.53878,-0.87424,1.8344$

$\mathrm{H}, 0,2.53878,0.87424,1.8344$

$\mathrm{H}, 0,3.02130,0 ., 4.1236$

$\mathrm{H}, 0,1.49563,0.88079,4.12997$

H, $0,1.49563,-0.88079,4.12997$

$\mathrm{C}, 0,-2.55353,0 .,-2.70104$

$\mathrm{H}, 0,-2.64852,0 .,-3.78498$

$\mathrm{H}, 0,-3.03987,-0.89101,-2.30578$

H, $0,-3.03987,0.89101,-2.30578$
Dipropyl sulfide

S, $0,1.04489,-0.40967,-0.4281968$

C, $0,0.8469,-0.53759,1.38001$

$\mathrm{H}, 0,1.7711,-1.00794,1.72099$

$\mathrm{H}, 0,0.83241,0.47192,1.79762$

C, $0,-0.36081,-1.34288,1.85692$

$\mathrm{H}, 0,-1.27914,-0.88331,1.48501$

$\mathrm{H}, 0,-0.31583,-2.34153,1.417$

C $, 0,-0.42195,-1.44220,3.38270$

C, $0,0.42195,-1.44220,3.38270$

$\mathrm{H}, 0,0.4693,-1.92938,3.78328$

, $0,-0.49365,-0.45493,3.84355$

$\mathrm{H}, 0,-1.28837,-2.02110,3.70492$

C, $0,-0.28491,0.76739,-0.85529$

$\mathrm{H}, 0,-1.25389,0.32872,-0.61362$

$\mathrm{H}, 0,-0.15788,1.66713,-0.24920$

C $, 0,-0.22504,1.11514,-2.34129$

C, $0,-0.3122,0.19846,-2.92954$

H, $0,0.75481,1.53912,-2.57244$

C, $0,-1.32441,2.09642,-2.75054$

$\mathrm{H}, 0,-2.31680,1.68345,-2.55960$

$\mathrm{H}, 0,-1.24403 .03514,-2.19895$

$\mathrm{H}, 0,-1.26302,2.33136,-3.81379$

Diisopropyl sulfide

$\mathrm{S}, 0,0.24402,-0.75054,-0.33339$

C, $0,0.23455,-0.3525,1.4643$

$\mathrm{H}, 0,1.02331,0.38765,1.61815$

C, $0,0.09810,0.90827,-1.13367$

$\mathrm{H}, 0,0.36656,1.63159,-0.36091$

C, $0,-1.09459,0.21321,1.9576$

$\mathrm{H}, 0,-1.35450,1.14387,1.45460$

$\mathrm{H}, 0,-1.90379,-0.49918,1.79170$

H, $0,-1.03786,0.42350,3.02915$

C, $0,0.6115,-1.63471,2.20574$

$\mathrm{H}, 0,-0.12987,-2.41706,2.03419$

$\mathrm{H}, 0,1.58251,-2.01085,1.88554$

$\mathrm{H}, 0,0.65404,-1.44507,3.27994$

C, $0,-1.32255,1.18671,-1.62481$

$\mathrm{H}, 0,-1.38302,2.18266,-2.07200$

$\mathrm{H}, 0,-1.6156,0.45936,-2.38332$

$\mathrm{H}, 0,-2.04884,1.13125,-0.81618$

C, $0,1.11355,1.00224,-2.27324$

$\mathrm{H}, 0,2.13196,0.87727,-1.9090$

$\mathrm{H}, 0,0.92895,0.23164,-3.02410$

H, $0,1.03788,1.9727,-2.7691$
Methyl ethyl disulfide

$\mathrm{S}, 0,-0.321159,-0.554920,-1.460754$

S, $0,-0.318710,-0.55790,0.591273$

C, $0,1.44593,-0.599284,-1.875215$

$\mathrm{H}, 0,1.505761,-0.651235,-2.955706$

$\mathrm{H}, 0,1.952764,0.293955,-1.537361$

$\mathrm{H}, 0,1.916391,-1.473518,-1.448757$

C, $0,-0.200776,1.215652,1.010777$

$\mathrm{H}, 0,0.725534,1.612657,0.616329$

$\mathrm{H}, 0,-1.022419,1.735132,0.536996$

C, $0,-0.252556,1.382091,2.5270$

H, $0,-1.180884,0.99626,2.933301$

$\mathrm{H}, 0,0.570367,0.866374,3.00969$

$\mathrm{H}, 0,-0.185199,2.434759,2.781580$

Diethyl disulfide

$\mathrm{S}, 0,-0.55906,-0.45534,-1.00286$

S. $0,-0.46464,-0.46363,1.0463$

C, $0,1.19982,-0.52944,-1.53514$

$\mathrm{H}, 0,1.71155,0.36225,-1.17670$

$\mathrm{H}, 0,1.65363,-1.4021,-1.0692$

$\mathrm{C}, 0,-0.32589,1.31377,1.49805$

$\mathrm{H}, 0,0.59756,1.7100,1.07894$

, $0,-1.16603,1.83962,1.04838$

C $0,1.25643,-0.6178,-3.05605$

$\mathrm{H}, 0,0.74633,-1.50929,-3.42193$

$\mathrm{H}, 0,0.79550,0.25232,-3.52444$

$\mathrm{H}, 0,2.29491,-0.66542,-3.3871$

C, $0,-0.33661,1.44373,3.01700$

$\mathrm{H}, 0,0.50041,0.91126,3.4696$

$\mathrm{H}, 0,-0.25779,2.49427,3.30063$

H, $0,-1.25927,1.04926,3.44345$
Ethyl tert-butyl disulfide

S, $0,-0.845753,-0.394027,-0.766337$ S, $0,-0.77808,-0.335282,1.285094$ C, $0,0.912956,-0.451792,-1.354254$ $\mathrm{C}, 0,-0.95867,1.441774,1.66979$ $\mathrm{H}, 0,-0.109688,1.982940,1.275790$ $\mathrm{H}, 0,-1.85092,1.807157,1.180040$ C, $0,0.781578,-0.460483,-2.883370$ H, $0,1.769971,-0.519504,-3.329417$ $\mathrm{H}, 0,0.20795,-1.314176,-3.227641$ $\mathrm{H}, 0,0.302133,0.442058,-3.246469$ C, $0,1.59735,-1.734579,-0.876814$ $\mathrm{H}, 0,2.609073,-1.778941,-1.272048$ $\mathrm{H}, 0,1.660288,-1.769750,0.204305$ $\mathrm{H}, 0,1.060690,-2.61302,-1.215973$ C, $0,1.701180,0.77773,-0.901018$ $\mathrm{H}, 0,1.225036,1.694764,-1.229576$ $\mathrm{H}, 0,1.802308,0.805680,0.177634$ $\mathrm{H}, 0,2.70190,0.7451,-1.32365$ C, $0,-1.05844,1.62108,3.18220$ $\mathrm{H}, 0,-1.17155,2.67415,3.41867$ $\mathrm{H}, 0,-1.91412,1.09133,3.58570$ $\mathrm{H}, 0,-0.16735,1.25871,3.68331$

Dipropyl disulfide

S, $0,0.03491,0.0666,0.0619$

$\mathrm{S}, 0,1.52534,-1.01759,0.96273$ C, $0,0.00871,-0.60696,-1.63874$ $\mathrm{H}, 0,0.97242,-0.42086,-2.09477$ $\mathrm{H}, 0,-0.13671,-1.67792,-1.58042$ C, $0,3.03067,-0.05791,0.57759$ $\mathrm{H}, 0,3.16421,-0.02261,-0.49652$ $4,0,28244,0.95403,0.93524$ C $0,-1.09794,0.04753,-2.47058$ $\mathrm{H}, 0,-0.97488,1.12686,-2.45264$ $\mathrm{H}, 0,-0.945920,-0.260363,-3.50183$ C, $0,4.242729,-0.70808,1.244653$ $\mathrm{H}, 0,4.343938,-1.731575,0.895548$ $\mathrm{H}, 0,4.081089,-0.757108,2.317324$ C, $0,-2.51710,-0.325219,-2.040246$ $\mathrm{H}, 0,-2.735715,0.009653,-1.03279$ $\mathrm{H}, 0,-3.245555,0.126628,-2.70576$ $\mathrm{H}, 0,-2.661711,-1.401165,-2.0708$ C, $0,5.530164,0.063957,0.95307$ $\mathrm{H}, 0,6.379900,-0.409097,1.434256$ H, $0,5.732579,0.103538,-0.113073$ $\mathrm{H}, 0,5.467698,1.084502,1.318434$ 
Dibutyl disulfide

$\mathrm{S}, 0,-1.258072,0.366890,0.380643$

S, $0,0.260353,-0.758749,1.178069$

C, $0,-1.365069,-0.249373,-1.33867$

$\mathrm{H}, 0,-0.414490,-0.070438,-1.82410$

$\mathrm{H}, 0,-1.53309,-1.318167,-1.309519$

C, $0,1.756352,0.201767,0.759355$

$\mathrm{H}, 0,1.841193,0.273683,-0.317791$

$\mathrm{H}, 0,1.643065,1,201395,1.157818$

C, $, 1.643065,1.201395,1.157818$

$\mathrm{C}, 0,-2.484384,0.457780,-2.10782$

$\mathrm{H}, 0,-2.336956,1.533786,-2.05603$

$\mathrm{H}, 0,-2.373929,0.184987,-3.155089$

C, $0,2.989823,-0.481976,1.348529$

$\mathrm{H}, 0,3.064951,-1.494219,0.959673$

$\mathrm{H}, 0,2.874817,-0.568463,2.42569$

C, $0,-3.900863,0.102867,-1.648055$

$\mathrm{H}, 0,-4.035944,0.383160,-0.608249$

$\mathrm{H}, 0,-4.029849,-0.976183,-1.6935$
$\mathrm{C}, 0,4.27704,0.28416,1.031239$

$\mathrm{C}, 0,4.27704,0.28416,1.031239$
$\mathrm{H}, 0,4.387853,0.373143,-0.047003$

$\mathrm{H}, 0,4.197587,1.297770,1.41688$

C, $0,-4.973793,0.780759,-2.499457$

H, $0,-4.891126,0.489842,-3.542991$

$\mathrm{H}, 0,-5.968895,0.512785,-2.15923$

$\mathrm{H}, 0,-5.968895,0.512785,-2.15923$
$\mathrm{H}, 0,-4.888121,1.862553,-2.45018$

$\mathrm{H}, 0,-4.888121,1.862553,-2.45018$
$\mathrm{C}, 0,5.519638,-0.386260,1.615991$

C, $0,5.519638,-0.386260,1.615991$
$\mathrm{H}, 0,6.416304,0.17651,1.376710$

$\mathrm{H}, 0,5.453151,-0.459515,2.697527$

$\mathrm{H}, 0,5.644491,-1.391228,1.223430$ 